\title{
"Je la salue, la conscience des hommes. " Le théâtre français de Malaparte et sa réception
}

"Je la salue, la conscience des hommes». Il teatro francese di Malaparte e la sua ricezione Oltralpe

"Je la salue, la conscience des hommes." Malaparte's French Theatre and Its

Reception

Filippo Fonio

\section{CpenEdition}

\section{Journals}

Édition électronique

URL : http://journals.openedition.org/cei/3448

DOI : $10.4000 /$ cei.3448

ISSN : 2260-779X

Éditeur

UGA Éditions/Université Grenoble Alpes

Édition imprimée

ISBN : 978-2-84310-370-4

ISSN : $1770-9571$

Référence électronique

Filippo Fonio, « « Je la salue, la conscience des hommes. » Le théâtre français de Malaparte et sa réception », Cahiers d'études italiennes [En ligne], 24 | 2017, mis en ligne le 28 février 2017, consulté le 28 mars 2021. URL : http://journals.openedition.org/cei/3448 ; DOI : https://doi.org/10.4000/cei.3448

Ce document a été généré automatiquement le 28 mars 2021.

(c) ELLUG 


\section{«Je la salue, la conscience des} hommes. » Le théâtre français de Malaparte et sa réception

"Je la salue, la conscience des hommes». Il teatro francese di Malaparte e la sua

ricezione Oltralpe

"Je la salue, la conscience des hommes." Malaparte's French Theatre and Its

Reception

Filippo Fonio

\section{Un Malaparte lecteur et visionnaire, dans l'ombre de D’Annunzio. Introduction}

1 Quelques mots sur le titre que j'ai choisi. Il s'agit de la réplique de clôture de Karl Marx dans la pièce de Malaparte Das Kapital ${ }^{1}$. Sortie de scène ô combien caustique de la part d'un personnage qui, tout au long de l'action dramatique, s'est vu tiraillé, non pas seulement par les autres - sa famille, les révolutionnaires, les exilés français (tout en étant exilé lui-même), Engels, ses autres collaborateurs, son secrétaire, Godson, et Dieu, même - mais aussi, et surtout, par sa conscience, dont sans doute Godson est une sorte d'hypostase...

D'autre part, la « conscience » peut être considérée comme une catégorie de lecture et d'analyse de l'œuvre de Malaparte particulièrement utile, même au-delà de son théâtre. D'autant plus que la prise en compte des "problèmes" et des évolutions de sa conscience d'homme et d'intellectuel peut sans doute nous fournir une grille de lecture diachronique de son œuvre, notamment des « ouvertures sur les autres et le monde " d'un côté, et du "repli sur sa région, ses proches, soi-même» d'un autre côté ${ }^{2}$. Le 27 janvier 1933 Malaparte écrivait de Londres à Daniel Halévy, au sujet de sa situation compliquée à l'égard du fascisme : 
Io deciderò secondo la mia coscienza. Parola dura, parola che non bisogna mai pronunciare, in Italia. Qualche volta io penso che la letteratura possa essere un rifugio, per gli italiani che hanno della coscienza, e hanno orrore di questa politica.

[...] Queste idee mi tormentano, e io non so dove sia la verità ${ }^{3}$.

3 Ce qui me semble intéressant dans cette prise de position est précisément l'idée de la littérature comme refuge, du désengagement de l'intellectuel - de l'Italien, nous dit Malaparte - comme seule solution pour rester fidèle à soi-même en des périodes qui font horreur, tout en ne risquant pas de mettre en danger sa liberté, sa vie, et tout en restant cohérent avec sa propre pensée et ses convictions. Et pour l'écrivain, ce hameau de paix, cette manière et cette méthode de ne pas trahir sa conscience coïncident avec la littérature. Dans le cas de cette confidence de Malaparte à un intellectuel qui lui était proche, «littérature » signifie non seulement l'écriture comme forme d'éloignement d'une politique qui effraie, mais également, et surtout, la lecture et l'idée d'un " nowhere », ou d'un " anywhere » que la lecture peut contribuer à créer. On sait bien, d'ailleurs, que la conduite de Malaparte ne s'est pas conformée à cette possibilité de sortir du dilemme de la conscience, si ce n'est que très épisodiquement. À ce propos, il faut considérer le théâtre comme l'une de ces - assez rares, tout considéré circonstances dans lesquelles Malaparte a décidé de s'offrir un répit et une pause par rapport à l'engagement, à la dénonciation ou à l'auto-apologie. La création dramaturgique serait l'une des circonstances qui l'ont vu servir la littérature, plutôt que se servir de celle-ci, l'une des circonstances, enfin, où il a décidé de "lâcher prise ", $\mathrm{du}$ moins partiellement, et de confier à quelqu'un d'autre l'aboutissement de projets forcément incomplets, comme le sont les pièces - même celles achevées - lorsqu'elles passent dans les mains d'une compagnie théâtrale et d'un metteur en scène.

C'est ainsi que les deux pièces «majeures » de Malaparte écrivain français, Du côté de chez Proust et Das Kapital, sont deux œuvres où le profil d'un Malaparte lecteur des classiques apparait de manière frappante, et son attitude face aux hypotextes se teint d'humilité, et presque de timidité, son appropriation des modèles est inspirée d'un grand respect. Malaparte veut s'offrir au lecteur et au spectateur, de Proust et de Marx, mais aussi de Malaparte lui-même, à la fois comme un fin exégète de l'œuvre d'autrui, et comme un écrivain qui re-réfléchit sur sa propre œuvre passée et qui planifie celle à venir.

5 La question du Malaparte lecteur me semble l'une des moins explorées jusqu'à présent. Si en effet les articles de presse, les reportages de guerre, les essais politiques mettent en évidence une composante, ou matrice, politico-mémorialiste et de témoignage, alors que la plupart de ses proses narratives - ses recueils de récits et ses romans, excepté sans doute Don Camalèo ${ }^{4}$ - se prêtent à une lecture fortement marquée par une pratique de l'autofiction, certains passages de celles-ci suggèrent par contre l'idée d'un Malaparte auteur livresque, lié à la tradition littéraire occidentale et aux monuments qui la constituent. Et le théâtre, notamment les deux pièces abouties, fait décidément pencher la balance en faveur d'un type de littérature intertextuelle et en même temps visionnaire, où la source est personnalisée et fait l'objet d'une appropriation qui ne la dénature pas pour autant. Malaparte auteur de théâtre se situe ainsi dans le cadre d'une tradition de relecture-adaptation fortement originale, visant à une sorte d'cemulatio interprétative.

6 La « conscience » d'une nécessité de l'engagement semble néanmoins refaire surface là où l'écrivain-lecteur tente de la refouler, la méditation sur les classiques se voyant dépassée par le besoin d'un retour sur le devant de la scène - sans laisser que les 
comédiens incarnent, mettent en bouche et en espace le fruit de ces lectures des classiques: d'où, probablement, l'abandon, à des phases de concrétisation différentes, de la plupart des programmes d'écriture dramatique de la part de Malaparte. Ces projets inachevés gardent néanmoins une importance non négligeable dans le cadre du développement de l'écriture malapartienne, précèdent ou parfois accompagnent le travail sur les pièces abouties et portées à la scène, en représentent des possibilités non réalisées et des hypothèses alternatives qui se sont révélées moins fécondes. Voire, elles constitueront les pièces du dossier génétique d'autres expériences d'écriture, notamment du théâtre en italien - dont la réalisation principale, et le projet de plus longue durée est constitué par Anche le donne hanno perso la guerra.

7 Parfois, le Malaparte témoin et auteur d'autofiction - intellectuel engagé - rattrape en quelque sorte le Malaparte lecteur et auteur dramatique, le Malaparte qui s'adonne à la contemplation et à la méditation. Ainsi, le public et les critiques - notamment la presse française - sont là pour lui rappeler que le buen retiro de la réécriture du classique n'est pas suffisant pour faire oublier les exploits « anti-quelque chose » des autres Malaparte. Lorsque ce n'est pas lui qui lance des battibecchi, il se devra de les subir. A fortiori, la réception française des deux pièces que Malaparte porte à la scène le mettra face à ses impossibilités et à son impuissance : il ne lui est pas permis d'écrire en français pour la scène parisienne - bien que ses admirateurs soient nombreux, y compris dans le milieu théâtral -, il lui est fortement déconseillé de se mesurer avec Proust, on lui tiendra grief d'avoir voulu écrire une pièce sur Marx qui ne soit ni marxiste ni antimarxiste. Bref, les « fours » de Du côté de chez Proust et de Das Kapital renforceront chez Malaparte le sentiment d'être persona non grata en France - plus que celui de ne pas être fait pour le théâtre - et sur ce fond d'amertume la " conscience » qui le conduira de nouveau à privilégier le témoignage et l'autofiction refera surface.

8 J'ai l'impression que Malaparte s'est pour le moins inspiré, du point de vue de ce profil de lecteur-visionnaire, d'un illustre prédécesseur, auquel il a d'ailleurs été comparé à plusieurs reprises par la critique tout comme par ses contemporains: Gabriele D'Annunzio. D'Annunzio est un écrivain - et un homme - que Malaparte « toute sa vie [...] a imité à sa manière ${ }^{5}$ ", et nombreux sont les points de contacts, presque les concomitances, entre les deux : ils ont été les élèves du même institut, le prestigieux lycée Cicognini de Prato, où Malaparte est entré à une époque où le mythe de l'ancien élève devenu célèbre hantait déjà l'établissement ${ }^{6}$. Ils nous ont laissé tous deux des pages merveilleuses sur la Toscane, sur ses paysages et ses habitants, sur le genius loci toscan pour ainsi dire ${ }^{7}$, et en même temps, ils ont vécu tout au long de leur carrière un engouement pour la France et la langue française. En outre, ils ont nourri un amour sans bornes pour leurs chiens ${ }^{8}$, ils ont pratiqué plusieurs formes et genres littéraires - tous, pourrait-on dire, du roman à la nouvelle, à la poésie, au théâtre et au scénario pour le cinéma, au journalisme, à la critique littéraire et la chronique de guerre, au journal intime, aux genres plus spécifiquement politiques - ils ont été tous deux des "poètes soldats ", ils ont été accusés de transformisme et d'opportunisme politique à maintes reprises et par des adversaires de tout horizon, ils ont eu des rapports controversés avec le fascisme et son chef de file, nous ont laissé chacun un «testament architectural », respectivement dans le Vittoriale et la Villa Malaparte, que Maurizio Serra définit, avec beaucoup d'à-propos, un « Controvittoriale 9 ".

De même que le style des deux auteurs, ainsi leurs poétiques présentent de nombreux points communs - ce que la réception de la part de la critique n'a pas manqué de 
souligner. On a souvent transposé au cas de Malaparte des étiquettes et des définitions qui avaient été créées pour D'Annunzio: son style, notamment celui des romans "majeurs ", par son décalage entre la dimension esthétique et la dimension éthique, rentre à plein titre dans le concept d'" amore sensuale della parola » que Mario Praz avait employé pour caractériser celui de D'Annunzio dans son La carne, la morte e il diavolo nella letteratura romantica (1938); l'on songe encore à Eugenio Montale, qui définira, en 1957, Malaparte - sur le plan existentiel et non pas littéraire cette fois-ci un "dilettante di sensazioni ${ }^{10}$ ", comme Benedetto Croce avait étiqueté D’Annunzio quelques décennies auparavant.

Dans les paragraphes qui suivent, on cherchera à illustrer ce parcours d'un Malaparte "lecteur» et "visionnaire" à travers les différentes étapes de son travail dramaturgique français, et ce en trois moments : tout d'abord, par un regard sur les projets théatraux inachevés, ensuite par un examen des deux pièces accomplies et portées à la scène, enfin par un approfondissement des raisons qui ont conduit à l'échec de ces textes lors de leur mise en scène.

\section{Projets inaboutis}

11 Tout au long de sa carrière d'écrivain, Malaparte a entamé l'écriture d'une dizaine de pièces, en italien et en français. La moitié de ces textes est restée inaccomplie.

Ces deux constats montrent à la fois que l'écriture pour le théâtre n'était pas un intérêt périphérique pour Malaparte, et que ce pan de son activité lui tenait particulièrement à cœur, car maints de ces textes montrent en effet que l'écrivain est revenu à plusieurs reprises sur certains projets - en particulier sur celui du «cycle» ou de la «série» Anche le donne hanno perso la guerra - tout en les ajustant, en les modifiant, en les abandonnant aussi lorsqu'il lui a semblé que la portée dramatique de ces scénarios n'était pas suffisante pour qu'ils accèdent à la scène ${ }^{11}$. L'épreuve de la scène était en effet un élément crucial pour Malaparte, homme de théâtre à sa manière, même si dépourvu d'une réelle expérience «technique » du théâtre, et spectateur assidu à ses heures ${ }^{12}$. Grand connaisseur des codes, et des modes du théâtre, sans aucun doute. C'est ainsi qu'il fréquente plusieurs types d'écriture théâtrale lors de ses expériences dans ce domaine, de l'avant-spectacle et la revue de variétés dans le cas de son dernier opus, Sexophone, à la comédie de mœurs dans Les Femmes de Sienne et, dans une certaine mesure, dans Du côté de chez Proust, au théâtre politique dans les cas des Joyeux compères de Yalta et - entre autres aspects - de Das Kapital, à l'allégorie historique dans le cas des Amazones.

13 Particulièrement riches et foisonnantes sur le plan générique sont les ébauches des pièces françaises inachevées, dont nous pouvons lire les fragments dans les volumes édités par Edda Ronchi Suckert au cours des années 1990. Tout ce matériel préparatoire - sujets de pièces, plans, indications de décors et costumes, et même des scènes et de actes de drames complets en premier jet, ainsi que le peu de pages que le Malaparte critique consacre au théâtre (y compris à sa propre œuvre) - donne l'impression que, tout comme D'Annunzio, encore une fois, Malaparte a été hanté par la «tentation du théâtre ». Les deux écrivains, à une époque où leur réputation était déjà suffisamment affirmée, ont cherché sur la scène un succès ultérieur et sans doute plus large, mais aussi, pensaient-ils, plus à portée de main. En relevant le défi que l'épreuve de la scène leur proposait, tous les deux ont constaté que ce n'était pas si simple que cela... 
14 Et même si le théâtre italien de D'Annunzio a été célèbre à son époque, et a en effet contribué à consolider sa renommée internationale (grâce notamment aux comédiennes qui se le sont approprié, de Sarah Bernhardt à Eleonora Duse, ou à Irma Gramatica), son théâtre français, nonobstant Ida Rubinstein, a eu des échos beaucoup plus mitigés, et aujourd'hui sa production dramatique dans les deux langues - à l'exception de La Figlia di Iorio - est tombée dans l'oubli. Un destin, une fois encore, commun avec Malaparte, qui, excepté sa pièce italienne Anche le donne hanno perso la guerra, n'a connu, lui, qu'un succès d'estime, et ce dès la création de ses pièces. De plus, les deux écrivains se sont mesurés avec la scène française - parisienne, qui plus est en écrivant eux-mêmes leurs textes français, ce qui a suscité la colère et même le mépris de plusieurs chroniqueurs et animateurs du "tout Paris»-de celui des années 1910 dans le cas de D'Annunzio, et de celui du deuxième après-guerre dans le cas de Malaparte.

15 Outre les deux pièces achevées et portées à la scène, dont il sera question au paragraphe suivant, la « série » du théâtre français de Malaparte compte en particulier deux projets d'écriture qui ont été abandonnés, mais qui méritent d'être relus ${ }^{13}$. Tous les textes théâtraux inaccomplis peuvent être inclus dans un seul et même projet plus ample, celui d'un cycle sur les femmes qui auraient gagné - ou perdu, selon les moments de la gestation malapartienne - la guerre. Les femmes et la guerre, ou l'après-guerre, sont donc au centre de ces brouillons dramatiques - comme de maints passages de Kaputt et de La pelle, d'ailleurs - sans que Malaparte arrive cependant à cibler son sujet de manière précise. Non seulement, en effet, les cadres historiques et géographiques de ces expérimentations dramatiques sont variés, mais les ébauches, aussi, nous permettent de comprendre que Malaparte a toujours conçu ces projets comme peu contraignants sur le plan spatio-temporel, et ce à travers une sousdétermination des détails et des possibilités de décontextualisation de l'action prévues dès le début. Évidente est donc la portée universelle de la tentative, de la part de Malaparte, d'approfondissement théâtral de la thématique des "femmes et la guerre ». Le caractère fluctuant $\mathrm{du}$ projet paraît d'autant plus manifeste si on compare les ébauches à la seule pièce accomplie de ce cycle, et pour ainsi dire éponyme, intitulée en effet Anche le donne hanno perso la guerra ${ }^{14}$. On ne peut pas vraiment affirmer que les projets inaboutis aient constitué un réservoir de matériel qui sera ensuite réélaboré dans cet autre texte. Chaque pièce ou fragment de pièce est en effet autonome, et, s'il faut chercher un élément de continuité, il faudrait sans doute l'identifier dans ce concept de fond, dans cette étude de la «phénoménologie féminine en temps de guerre ", qui sera au centre des intérêts de Malaparte même au-delà du théâtre. Les deux ébauches remontent aux années 1947-1949, elles précèdent donc de quelques années le volet italien du « cycle ».

\section{Les Amazones ${ }^{15}$}

Les Amazones, drame ébauché en 1947 et interrompu au cours de l'acte II, est une sorte de rêverie post-hitlérienne - l'action se situe environ cinquante ans après la mort d'Hitler, à Rome - dans laquelle les Amazones, les seules femmes qui soient capables de s'opposer à la toute-puissance masculine hégémonique en Occident, décident de partir à la conquête de l'Europe. Au cours des scènes écrites pas Malaparte on assiste aux dilemmes des personnages masculins - le Roi et le commandeur en chef de son armée - par rapport à l'opportunité et à la légitimité de guerroyer contre des femmes. 
Une fois dépassés leurs scrupules "de genre ", la bataille entre les deux camps nous est racontée par un héraut, et les Amazones victorieuses cherchent à bâtir une politique « gynocentrique » dans leur nouveau fief.

Parmi les aspects des Amazones qui sont les plus intéressants, en ce qu'ils préludent au théâtre successif, on peut remarquer l'insistance sur les détails des costumes des personnages. Ainsi, dans cette pièce "d'anticipation ", nous trouvons le général en chef des armées royales, Alexandre Farnèse, qui entre en scène «[...] tout fermé dans son armure d'argent, qu'un maître italien du $\mathrm{XVI}^{\mathrm{e}}$ siècle a dessinée et créée pour quelque personnage d'un théâtre des $\mathrm{Pupi}^{16}{ }^{16}$. Pour décrire son costume, Malaparte fait référence dans une longue didascalie - sur le modèle en particulier de celles qui seront dans $D u$ côté de chez Proust - aux fresques de Pompéi et au mythe d'Icare, en créant ainsi une superposition d'époques à travers des références artistiques et culturelles. Il se sert également de l'ekphrasis, là où il précise que «sur la poitrine d'argent [de l'armure de Farnèse] sont sculptés les épisodes célèbres des amours de Mars et Vénus ${ }^{17}$ ».

Les costumes des Amazones sont conçus avec le même soin. Celles-ci, jeunes femmes ravissantes, portent, en bataille, des "masques de la Vieillesse », et leur Reine Ippolita " est habillée comme les hérö̈nes du Tasse dans les gravures du XvIII ${ }^{\mathrm{e}}$ siècle ${ }^{18}$ ". Le masque de la Reine des Amazones est tout particulièrement affreux : "[Il] représente un visage de vieille femme, sorcière ou ogresse, crevassé de rides profondes, dans lesquelles un sang boueux coule lentement [... $]^{19}$. C Ce qui n'empêche ni le syncrétisme habituel, ni la référence culturelle, car le même personnage est comparé plus loin à « une amazone des temps de Musset $^{20}$ ».

19 Le Roi de cette ville de Rome envahie par les Amazones, quant à lui, se revêt d'une armure qui est « un costume de rugby américain ${ }^{21}$ ». Et la bataille est également décrite sur le mode du commentaire d'un match de rugby. En voici la chronique à travers les mots du Grand Chambellan :

Le Roi a percé! Il passe à droite, renverse deux joueurs du team adversaire, on l'arrête, on l'enlace, on se jette à ses jambes, on arrache ses jambières, on tâche de le renverser. Mail il se dégage d'un coup d'épaule, renverse dans la boue ses adversaires, perce, sort en trombe du package, il tackle, il hésite, il cherche des yeux un passage au milieu de l'effroyable scrimmage des ennemies, il voit la Reine des Amazones, il la reconnaît à son armure, à son plumage, il court sur elle, la rejoint, bouscule son cheval d'un coup d'épaule, le cheval tombe, la Reine se relève, lève l'épée, reconnaît le Roi, jette l'épée, le Roi l'enlace de ses deux $\operatorname{bras}^{22}$...

20 Le mélange des époques et des styles, ainsi que l'imbrication de références culturelles s'imposent aussi dans les décors conçus par Malaparte. La salle des banquets du palais royal est décorée en accord avec ce principe :

Aux murs pendent des tableaux de l'ancienne et de la moderne école italienne, alternés avec grâce et ironie : des guerriers empanachés et des chevaux galopant de Giorgio De Chirico, des portraits de Sebastiano del Piombo à côté d'un paysage de Filippo De Pisis, une nature morte vénitienne à côté d'une nature morte de Giorgio Morandi, un tableau de bataille de Salvator Rosa à côté d'une bataille de Aligi Sassu, un Polichinelle de Magnasco à côté d'un Arlequin de ${ }^{* 23}$.

\section{Les Femmes de Sienne}

21 Les Femmes de Sienne ${ }^{24}$ se situe de manière encore plus marquée dans le cadre du cycle des femmes qui ont perdu (ou gagné) la guerre. Cette autre ébauche de pièce en trois actes $^{25}$ a été écrite par Malaparte à Paris au cours de février 1949, pendant celle qu'on 
peut considérer comme la période la plus intense de travail sur le théâtre français de la part de l'écrivain ${ }^{26}$. Des éléments de continuité entre Les Femmes de Sienne et les autres projets dramatiques français de Malaparte sont aisément reconnaissables. Ainsi, Malaparte aurait-il pensé à «ses » comédiens, Pierre Fresnay et Yvonne Printemps ${ }^{27}$, respectivement pour les rôles de Messer Toso de' Tolomei - originairement Toso degli Albizzi - et de Monna Beatrice ${ }^{28}$.

Cela dit, Les Femmes de Sienne se caractérise également par une série d'éléments qui la distinguent du reste du corpus théâtral malapartien. Il s'agit en effet de la seule œuvre dramatique de l'écrivain qui aurait sans doute dû se présenter comme une "comédie " - eût-elle été achevée. Tant le style de l'ébauche, en effet - «brillant» et dépourvu des tirades qui alourdissent parfois les autres pièces de Malaparte, et surtout basé sur la repartie rapide, la boutade, l'équivoque ${ }^{29}$ - que l'action elle-même, dont le noyau est une sorte d'échange marchand de marque boccacienne entre femmes et nourriture, font des Femmes de Sienne l'expérience dramatique sans doute la plus «légère » du Malaparte français. Les Femmes de Sienne se démarque des autres projets théâtraux aussi du fait qu'il se situe dans un cadre historique - le village fortifié de Montelsa, près de Sienne, en $1530^{30}$, à l'époque du siège de la ville par l'armée espagnole de Charles Quint. L'aspect proprement historique doit néanmoins être conçu de manière assez large, et peu contraignante, nous précise Malaparte: «Bien qu'elle se passe au XVI ${ }^{\mathrm{e}}$ siècle, en Italie, les costumes de la pièce sont modernes : $1890^{31}$.» Et encore :

Il ne faut pas trop s'attarder sur son costume [celui de Messer Vanni, le chef de file du parti adverse à celui de Messer Toso, et le premier personnage sur scène au lever du rideau], ses manières, son regard, ses paroles [...] : car si l'action se situe en 1530, il y a bien des choses dans la pièce, et même dans le personnage de Messer Vanni, qui montrent que l'action pourrait se passer n'importe quand ${ }^{32}$, même de nos jours ${ }^{33}$.

L'image sous-jacente au texte, et pour ainsi dire la métaphore qui le porte, est l'identification entre la ville et la (vertu de la) femme, les deux pouvant faire l'objet d'un siège de la part d'assaillants "étrangers ». Ce qui paraît manifeste dans une réplique de Monna Vanna, la femme de Messer Toso, adressée à sa belle-sœur, Béatrice, lorsque cette dernière est sur le point de partir pour le campement des assiégeants où elle passera la nuit : «Si l'ennemi se fait trop pressant, s'il se rapproche un peu trop des remparts qui défendent la citadelle de ta vertu, s'il menace d'ouvrir une brèche dans les murs de ta pureté, si les forces te manquent, ou si tu n'as plus envie de te battre, eh bien, dans ce cas, il faut se rendre ${ }^{34}$.» Cette citation montre bien que Les Femmes de Sienne se rapproche du cynisme politique que Malaparte révèle dans maints de ses autres textes, car le reniement de la patrie, des maris, de la fidélité conjugale de la part de ces femmes " assiégées » s'avère être un avantage pour la patrie elle-même - outre la distribution de vivres, dans la proportion d'un sac de blé et d'une pièce de bétail pour chaque femme qu'on laisse sortir la nuit, les Espagnols, une fois leur «désir de tendresse " assouvi, décident de lever le siège, et la pièce aurait dû se terminer sur les fiançailles entre la sœur du Podestat de Montelsa et un notable espagnol-, mais se concrétise aussi en une jouissance féminine (pour le sacrifice accompli?) qui ne manque pas de semer la panique au sein de la gent masculine.

Rien que dans ces ébauches paraît évident le caractère antithéâtral qu'on retrouvera dans les projets aboutis de Malaparte. Encore une fois, il s'agit d'un aspect commun à Malaparte et à D'Annunzio. Si, en effet, les deux écrivains avaient indéniablement un sens de la vie "théâtral » et " théâtralisé » très poussé, à cela ne correspondait pas un 
sens de la scène, une disposition, naturelle ou réfléchie, à l'écriture théâtrale... Le théâtre de Malaparte n'est pas antithéâtral au sens où on définirait ainsi celui de Beckett, ou de Pirandello par exemple. Malaparte ne tente pas de miner de l'intérieur les structures de la forme-théâtre, en travaillant, en tant que connaisseur des dynamiques profondes du texte dramatique, à la refonte d'un genre et au renouvellement d'une tradition. Son intérêt n'est pas non plus porté à la composante méta-théâtrale, comme cela est très fréquent à la période où se situent ses expériences dramatiques. Il ne s'intéresse pas, en effet, à éclaircir, ni à déconstruire les dynamiques internes des métiers et du monde du théâtre, l'establishment des spectacles, les systèmes économiques ou artistiques qui les régissent. Malaparte et D'Annunzio cherchent tous deux, par contre, à se conformer aux conventions du texte dramatique, sans pour autant dénaturer leur style de dramaturges non professionnels, et c'est plutôt là que réside le noyau problématique de leur rapport face à ce type très particulier de texte qu'est le texte pour la scène. Leurs tentatives d'écrire du théâtre, ou d'écrire pour le théâtre, échouent en grande partie - d'où, probablement, le nombre important de projets dramatiques laissés inachevés par Malaparte tout comme par D'Annunzio. Et si tous deux sont des auteurs de "théâtre dans un fauteuil", les pièces de Malaparte souffrent tout particulièrement d'un certain nombre de défaut "antithéâtraux » : une absence de rythme dans l'enchaînement des répliques, des longues didascalies très peu fonctionnelles et plus suggestives que "techniques", des indications de scène très difficiles à suivre - ainsi, vers le début de l'acte II de Das Kapital, par exemple, une didascalie précise qu'Engels dit sa réplique en «Exprimant, à peu près avec les mêmes mots, les idées qu'un retrouve dans le Manifeste de 1848, dans ses lettres de 1851 à Marx et dans sa Contribution à l'histoire de la Ligue communiste ${ }^{35}$ ». De plus, la composante que j'ai défini «livresque " et "visionnaire » du théâtre de Malaparte, et de celui de D'Annunzio, éloigne ultérieurement les réalisations dramatiques des deux auteurs des différentes formes d'écriture pour le théâtre, de celles plus traditionnelles tout comme $\mathrm{du}$ théâtre avant-gardiste et expérimental. Ces caractères sont d'ailleurs encore plus manifestes dans les pièces achevées.

\section{Projets achevés ${ }^{36}$}

Du côté de chez Proust et Das Kapital ont été écrits très rapidement (en l'espace de quelques mois), à la même époque et presque simultanément, directement en français. Il semblerait que Malaparte n'ait pas eu recours à une relecture par des amis ou collaborateurs francophones - ou du moins qu'il n'ait pas corrigé son texte suite aux remarques de ses premiers lecteurs ${ }^{37}$.

Il est utile tout d'abord de préciser quelques détails techniques sur les deux pièces, avant de passer à un examen plus détaillé de chaque texte.

Du côté de chez Proust, « impromptu en un acte avec musique et chant ", est créé à Paris au théâtre de la Michodière le 22 novembre 1948 par la mise en scène de Pierre Fresnay, qui incarne également Marcel Proust dans la pièce ${ }^{38}$. Il reste à l'affiche pour un nombre de représentations qu'il est très difficile de préciser, mais qu'on peut supposer avoir été assez consistant ${ }^{39}$. Il a été publié pour la première fois dans le supplément théâtral de la revue Opéra en mars $1949^{40}$. Il a aussi été traduit en italien pour la scène par Enzo Ferrieri, cette version demeurant inédite ${ }^{41}$. Malaparte aurait réalisé une commande qui lui avait été faite de la part de la compagnie Fresnay-Printemps, et la forme brève de la 
pièce est due au fait que $\mathrm{Du}$ côté de chez Proust a été conçu comme après-spectacle aux CEufs de l'Autruche d'André Roussin, toujours mis en scène par, et avec Pierre Fresnay en tête d'affiche ${ }^{42}$.

Das Kapital, pièce en trois actes, est créé au théâtre de Paris le 29 janvier 1949 par la mise en scène de Pierre Dux, qui interprète aussi Marx dans la pièce après le refus de Michel Simon ${ }^{43}$. La pièce serait restée à l'affiche pendant pas moins de quatre-vingt représentations ${ }^{44}$. Das Kapital a été publié pour la première fois dans le supplément théâtral de la revue France-Illustration en juin $1949^{45}$ et en volume chez Denoël l'année suivante, précédé par Du côté de chez Proust. La princeps italienne est de 1951.

\section{Du côté de chez Proust ${ }^{46}$} première partie du roman porte le titre Le Côté de Guermantes, et contient en particulier une touchante réévocation du Paris proustien ${ }^{49}$ - des extérieurs proustiens - qui est complémentaire à celle des intérieurs proustiens dont le décor de Du côté de chez Proust représente une sorte de summa. Et le " côté Guermantes " revient d'ailleurs à plusieurs reprises dans Kaputt, avec ses paysages, sa "fatigue ", ses figures fanées par le temps passé et comme balayées par la guerre ${ }^{50}$, alors que, à travers une analepse qui le fait remonter jusqu'en 1905, dans Du côté de chez Proust Malaparte peut approfondir l'étude de la société proustienne, en analysant une époque où sont jetées les bases de la désagrégation sociale qui sera un fait accompli à la période où se situe l'action de Kaputt. off » de la Recherche, tout comme Das Kapital le serait par rapport à l'ouvrage de Marx, met en scène la première rencontre entre deux personnages proustiens, Rachel Quand du Seigneur et Robert de Saint-Loup, que Malaparte situe «autour de 1905 » dans la garçonnière de Robert, et qu'il imagine être advenue en la présence de Marcel Proust lui-même. C'est là un parti pris d'une certaine inventivité de la part de Malaparte, lorsqu'il décide de "donner corps» au principe autobiographique qui régit la construction proustienne.

33 La scène est meublée en guise d'un « intérieur proustien, où tout est Guermantes ${ }^{51}$ ", ce qui, dès la didascalie initiale très détaillée, alerte le lecteur, tout comme le spectateur proustien de l'époque, du fait qu'il s'agit d'une œuvre manifestement «au second 
degré ». Or, Proust est considéré par Malaparte comme l'intellectuel symbole de la crise de la génération de la fin du siècle, l'incarnation d'une littérature de la décadence qui prélude au désagrègement de la société qui sera décrit dans Kaputt ou dans La pelle; mais Proust est en même temps le peintre par excellence de la société bourgeoise (en étant lui-même bourgeois) - une société dont l'immobilisme et le déterminisme social de la Recherche seraient la parfaite représentation. De plus, l'approche de Malaparte à la Recherche est foncièrement marxiste, comme cela est clairement affirmé dès la didascalie initiale - ce qui n'a pas manqué d'agacer le public parisien, comme on le verra. La décadence du faubourg Saint-Germain mise en scène par Malaparte, suite à une lecture fortement subjective et «malapartienne » de la Recherche, est ainsi non pas tant le fruit de la dégénérescence des mœurs des classes privilégiées, que le fruit de « la fatalité des lois de l'évolution sociale ${ }^{52} »$. La corruption des mœurs serait en effet le symbole de cette dégénérescence, plus que sa cause.

Le décor conçu en détail par Malaparte pour son acte unique participe de cet air de " fin de siècle ", il est lui aussi décrit en accord avec les stylèmes de la Décadence, aux intérieurs de la Recherche se superposant ceux d'À rebours et de maintes œuvres de la même teneur. L'on y trouve ainsi les permutations coutumières entre nature et art, d'après une conception qui était déjà celle de Pater et de Wilde - ou de Théophile Gautier, pour la France -, selon laquelle ce serait la première qui imiterait le deuxième. Malaparte se situe donc dans une esthétique suggestive, et non pas imitative ${ }^{53}$. Dans la constante mise en abîme en laquelle consiste $D u$ côté de chez Proust, aux murs de la garçonnière de Robert sont accrochés des tableaux représentant des personnages de la Recherche, mêlés à d'autres portraits des intimes de Proust, tel Robert de Montesquiou, qui y sont transfigurés en personnages de roman. Les citations du cycle proustien - parfois avec références précises, parfois plus indirectes - abondent du reste dans la didascalie initiale comme dans le reste de la pièce.

Comme on l'a déjà observé par rapport aux projets théâtraux inaboutis, le côté synoptique de plusieurs éléments du décor tend à abolir la distance entre les époques, à revendiquer une doctrine de l'éternel retour des coutumes et des modes, des styles et des sujets artistiques : le mannequin portant un habit de Fortuny conçu sur le modèle de la Renaissance vénitienne ${ }^{54}$ se combine à une silhouette qui renvoie à la série des villes de Giorgio De Chirico - qui seront réalisées à des années de distance par rapport à l'époque où est située l'action de la pièce. Le mannequin est «le portrait métaphysique des Guermantes ${ }^{55}$ ». De même, les canéphores du décor sont des vierges préraphaélites, les fauteuils sont apparentés à ceux conçus par les surréalistes. Outre cette superposition de modes et de référents, une hantise littéraire caractérise ce décor: la bergère qui trône sur la scène est empruntée à un roman de Louise de Vilmorin, les porcelaines sont celles des opérettes viennoises. Tout cela témoigne de « l'exquis mauvais goût de cette génération proustienne [...] que Marcel Proust appelle "littéraire" ${ }^{56} "$.

Les deux personnages proustiens que Malaparte choisit de mettre en scène, Robert de Saint-Loup et Rachel Quand du Seigneur, tout en gardant une composante de représentativité emblématique, respectivement, de l'aristocratie et du tiers état, seraient assimilés sur le plan de l'appartenance commune à la génération des " uraniens ${ }^{57}$ ", des adeptes du "vice sacrés8 », des "invertis ${ }^{59}$ », et seraient, dans le même temps, les représentants de la première génération marxiste, caractérisée par « le pressentiment du rôle que l'homosexualité allait jouer dans la désintégration de la 
société capitaliste ${ }^{60} »$. L'inversion sexuelle était, pour les intellectuels sympathisant du socialisme de la génération de Robert de Saint-Loup ${ }^{61}$, un instrument - sans doute le principal, dans l'optique ici adoptée par Malaparte - de la lutte des classes, leur « nonalignement sexuel » contribuant à bouleverser l'ordre social préétabli ${ }^{62}$. Dans le monde représenté par Proust, "homosexualité aristocratique et «homosexualité prolétarienne ${ }^{63}$ » sont en attirance réciproque, comme le montre du reste en particulier le personnage de Charlus. C'est même grâce au «vice social» des prolétaires, alliés sur ce plan aux aristocrates qui partagent les mêmes "goûts", que la crise de la bourgeoisie se consommerait à l'époque de la Recherche ${ }^{64}$.

La caractérisation vestimentaire et verbale des trois personnages de Du côté de chez Proust s'aligne sur ce parti pris politique, s'en sert comme d'une démonstration - c'est pourquoi il serait légitime de considérer l'impromptu malapartien comme une pièce « à thèse ». Ainsi Marcel Proust, "l'air imposant et minable, tel qu'il apparaît dans les photos et dans les portraits ${ }^{65}$ ", au gros mouchoir auquel il a constamment recours - ce qui donne lieu à une suite de mouvements grotesques sur scène - incarne ici la bourgeoisie, autant sur le plan de l'appartenance de classe que sur celui de la chronique et de la représentation de cette même classe. Il se présente néanmoins dès l'abord comme étant à son tour le produit d'un brassage de peuples, il est caractérisé par des traits virils - un côté gaulois - et par d'autres féminins ou efféminés - un côté " grec de Smyrne $»^{66}$; Robert de Saint-Loup, ami du narrateur du roman, est un dandy accompli, soucieux de garder un équilibre entre une composante de séduction éminemment masculine - à l'égard de Rachel - et une camaraderie quelque peu équivoque face à Proust: "Son insolence, sa désinvolture sont à la fois viriles et efféminées $^{67}$." Mais c'est une aristocratie nouvelle que celle de Robert relu par Malaparte, qui, au modèle figé par le dix-neuvième siècle, superpose un caractère décadent et maladif, emprunté aux aristocrates de Thomas Mann.

Enfin, Rachel Quand du Seigneur, " prolétaire campée dans le grand monde ${ }^{68}$ ", incarne ici le peuple, ce facteur de désagrègement des autres classes sociales. Elle se caractérise par un acharnement pour l'auto-affirmation, et par une absence de scrupules qui, selon Malaparte, lui garantiront la victoire dans la lutte entre l'ancien et le nouvel ordre social. La Rachel de Du côté de chez Proust est à la fois la " petite poule » d'À l'ombre des jeunes filles en fleurs et du Côté de Guermantes, et l'actrice qui remplace la Berma dans le gotha du monde du théâtre à la mort de la première, dont elle représente en quelque sorte un double en ton mineur, autant sur le plan social que sur celui du sublime artistique, dans le Temps retrouvé ${ }^{6}$. L'action de la pièce de Malaparte, qui se réduit à l'espace d'une soirée, cherche donc à englober la durée intergénérationnelle de la Recherche de Proust.

Rachel Quand du Seigneur et Robert de Saint-Loup sont accompagnés, à leur entrée en scène, d'une didascalie très détaillée sur l'habillement et le caractère des personnages, s'étalant sur plusieurs pages. Ces longues didascalies donnent d'ailleurs à Malaparte l'occasion d'aller bien au-delà d'une simple présentation "utilitaire »: elles ont un caractère métalittéraire évident. D'un côté, en effet, elles offrent au dramaturge la possibilité de fournir une clé de lecture du texte, et ce dès le début de celui-ci, à travers la grille sociétale marxiste déjà évoquée ; de l'autre côté, les denses renvois aux romans de Proust ne s'y limitent pas à des citations, mais ils s'étendent à une critique proustienne et même à une tentative d'«émendation» du texte de Proust ${ }^{70}$. Ainsi, Malaparte tente de rétablir, contre Proust, la vérité autour du personnage de Rachel 
Quand du Seigneur, qui aurait été victime de la jalousie de l'auteur-narrateur de la Recherche, et que Malaparte se propose de blanchir de tout ternissement. Au long de l'action scénique, Malaparte cherche à mettre en avant l'aspect "pythique ${ }^{71}$ " de Rachel, surtout face à un (micro-)univers masculin qui tend plutôt à la considérer comme « un jouet mécanique ${ }^{72} »$. Tandis que Proust remarque, lorsque Marcel et Robert se rendent là où Rachel habite :

[...] ce matin-là, et probablement pour la seule fois, Robert s'évada un instant hors de la femme que, tendresse après tendresse, il avait lentement composée, et aperçut tout d'un coup [...] une autre Rachel, un double d'elle, mais absolument différent et qui figurait une simple petite grue ${ }^{73}$.

Malaparte, au contraire, renverse ce jugement, et fait de Rachel un personnage triomphant et incarnant, sous les semblants d'une coquetterie de manière, une force vive de la société - la seule, sans doute. Et c'est là le vrai sujet de la pièce : une scène unique tirée d'un moment de la Recherche, qu'un lecteur de Proust pourrait considérer comme anodin, est "décortiquée " par le dramaturge qui en fait émerger la portée révolutionnaire, ou du moins fortement politisée. Rachel est au centre de la scène - et elle devrait l'être aussi au sein du système de la Recherche, suggère Malaparte, car "L'atmosphère où baignent, grâce à Rachel, tous les personnages proustiens est l'atmosphère même du marxisme $[. . .]^{74}$ ".

41 Si Proust, en effet, avait assisté à la première rencontre entre Saint-Loup et Rachel que Malaparte imagine, il n'aurait pu ne pas saisir le caractère emblématique de cette rencontre, sa nature allégorique pour ainsi dire, comme l'est forcément celle entre les représentants de deux «races» appelées à collaborer - ou à se détruire réciproquement - dans le nouveau monde que la Recherche inaugure tout en signant la fin de l'ancien. Le mépris dont les jugements de Marcel sur Rachel sont teints aurait sans doute été redimensionné, si le narrateur de la Recherche eût pu assister à la scène représentée dans Du côté de chez Proust ${ }^{75}$. Le Proust de Malaparte est le personnagecharnière de cette rencontre allégorique, éternellement en équilibre entre un amour nostalgique pour une aristocratie sur le point de disparaitre et incarnant les valeurs traditionnelles, et une incrédulité méprisante face à un prolétariat en ascension sociale, porteur de valeurs révolutionnaires. Il est le bourgeois finalement exclu tant par les uns que par les autres.

Même s'il n'y a pas d'action dramatique à proprement parler dans Du côté de chez Proust, ce qui fait percevoir au spectateur et au lecteur qu'une progression - et presque une "trame»- existe, et qu'on va vers le dénouement, c'est l'impression croissante d'échec éprouvée par Proust en tant que représentant et chantre de la bourgeoisie ${ }^{76}$. La passion de Robert de Saint-Loup et de Rachel métaphorise l'alliance entre les deux classes sociales dont ils sont les représentants : une alliance entre des forces destinées à écarter la bourgeoisie de la lutte politique.

Dans ce mélange d'une composante métalittéraire et d'une lecture politique de la Recherche réside sans doute l'aspect le plus intéressant de l'impromptu de Malaparte - son caractère « moderne ». De manière audacieuse, par exemple, la didascalie initiale de Du côté de chez Proust précise que les objets et meubles du décor doivent avoir

[...] le même rôle que les personnages de Marcel Proust : le rôle à la fois de témoins et de protagonistes de cette décadence de la société parisienne au début du siècle, dont les causes sont à rechercher non dans la corruption des mœurs mais dans la fatalité des lois de l'évolution sociale, étudiée par le marxisme ${ }^{77}$. 
44 Rachel et Proust, car on y trouve à maintes reprises une apologie de l'œuvre proustienne, une auto-défense de l'auteur face au point de vue des classes populaires qui prônent des instances moins intellectualistes, en art, dans le portrait de la société. Il n'est pas exclu, de ce point de vue, que Malaparte ait voulu, tout en parlant de Proust, prendre position par rapport au théâtre de Jean-Paul Sartre, dont les pièces se jouaient au même moment sur les scènes parisiennes.

Les longues didascalies semblent indispensables à la compréhension du texte, ce qui pourrait avoir posé problème lors de la création de la pièce à La Michodière. Les jeux de scène et les parties chantées par Yvonne Printemps ne sont sans doute pas suffisants pour combler la perte d'information due à l'absence des didascalies, qui suggèrent en outre la progression de l'action sur un mode d'accentuation du conflit entre les personnages/classes sociales. Le verbiage des personnages cherche à pallier ce manque, et il doit très probablement être considéré comme une stratégie de progression dramatique mise en place par Malaparte pour la version scénique. Il me semble en effet qu'aux dialogues de l'impromptu doit être attribuée une fonction qui va bien au-delà de la « réalisation souvent aphasique » constatée par Maurizio Serra ${ }^{78}$.

L'interprétation marxiste de la Recherche proposée par Malaparte, a contrario, véhicule une lecture de l'hypotexte proustien qui tend à en sous-dimensionner la portée érotique $^{79}$. Elle prélude en ce sens aussi à la deuxième pièce aboutie du théâtre français de Malaparte, dont la composition suit de près celle de Du côté de chez Proust : Das Kapital.

\section{Das Kapital}

Écrite par Malaparte à Paris en mai $1948^{80}$, Das Kapital est une pièce à la construction dramatique plus complexe. Il s'agit d'une comédie en trois actes, qui comptait, lors de la prépublication et de la création, vingt et un personnages, alors qu'il n'en reste que vingt dans l'édition définitive - même si la liste des dramatis personce continue d'en indiquer un de plus, sans doute à cause d'une inadvertance de la part de Malaparte. Parmi les rôles de la pièce, l'on trouve autant de figures historiques que de personnages inventés, c'est pourquoi il est légitime de considérer Das Kapital comme une « fiction historique $»^{81}$.

L'action dramatique, qui comme dans l'impromptu est située en un lieu unique - le salon de l'appartement de Marx - est localisée très précisément : à Londres, où réside Marx après avoir quitté Paris en 1849, et en l'espace de moins de vingt-quatre heures, précisément de l'après-midi du 3 décembre au 4 décembre 1851 à 10 heures du matin. Ces dates ne sont pas le fruit du hasard, car comme le rappelle dès le début la didascalie liminaire, on est au lendemain du « 18 brumaire de Louis-Napoléon [le 2 décembre 1851] "82 ", le coup d'État de Louis-Napoléon qui met fin à la Deuxième République, et qui entame le processus de restauration autoritaire qui aboutira au Second Empire.

Si, dans Du côté de chez Proust nous étions dans un « cas d'école » de la fiction littéraire postmoderne en quelque sorte, à savoir la rencontre entre un auteur et ses personnages, Das Kapital se situe plutôt dans le genre théâtral - et littéraire - de la «tranche de vie», celle de Karl Marx en l'occurrence, lors de son exil à Londres et pendant la rédaction du Capital. La structure de la pièce est plus classique que celle de l'impromptu proustien. La didascalie initiale suggère même une volonté anachronique

Cahiers d'études italiennes, 24 | 2017 
de respect des "trois unités » pseudo-aristotéliques. Malaparte ne déroge pas pour autant à sa tendance à la superposition des plans chronologiques, car l'on trouve parmi les personnages Felice Orsini, que Marx cherche à convaincre de ne pas «passer à l'acte » dans son projet d'attentat à Napoléon - qui aura lieu en janvier 1858. Ce jour dans la vie de Marx n'est pas seulement celui du coup d'État de Louis-Napoléon en France, mais aussi - avec un léger anachronisme - celui de la mort de l'un des fils de Marx, « Mush » (advenue en réalité quelques années plus tard).

La contextualisation des événements en concomitance avec le coup d'État de 1851, et le fait de définir ce dernier un "deuxième 18 brumaire " renvoie à l'œuvre écrite par Marx lui-même en 1852 sur Louis Bonaparte et son ascension au pouvoir ${ }^{83}$, qui constitue l'une des sources principales du travail de Malaparte. D'ailleurs, tout comme Du côté de chez Proust est tissé de citations de la Recherche, Das Kapital a plusieurs sources livresques, qui sont très présentes et le plus souvent signalées en didascalie: la correspondance entre Marx et Engels, et les lettres écrites par Jenny, la femme de Marx (tous trois sont parmi les personnages principaux de l'action dramatique), mais aussi de nombreuses citations du Capital et du Manifeste de 1848. Cette synergie entre hypotextes à caractère privé et œuvres publiées me semble emblématique du sens profond de Das Kapital. On dirait en effet que Malaparte a voulu montrer la genèse expérientielle et humaine des écrits de Marx - même les plus théoriques, tels le Capital-, étudier comment ses expériences existentielles, ici notamment la pauvreté et la souffrance des siens, la perte d'un deuxième enfant, ont influencé sa réflexion sur le rôle du prolétariat dans la révolution et sur l'opportunité même d'une révolution « authentique » - c'est-à-dire non pilotée par la bourgeoise ou l'aristocratie.

Le portrait que Malaparte fait ici de Marx est un portrait presque « en petit bourgeois » - un choix qui n'était pas pour plaire aux marxistes. Et c'est là d'ailleurs un sort auquel l'écrivain avait déjà destiné le théoricien du communisme dans ses écrits de la période fasciste, mais qu'il réserve également à Lénine dans Le Bonhomme Lénine. À quelques détails près: alors que le "bonhomme Lénine " est écrasé par les instances du prolétariat, le Marx de Malaparte, en vertu de sa conscience - qui n'est qu'accessoirement, dans Das Kapital, une conscience de classe, mais qui est surtout une conscience individuelle - arrive à tenir tête au prolétariat, la classe sociale dont il est le champion, le théoricien et - là encore, comme dans le cas de Proust - le chantre. Déjà en 1929, Malaparte s'était penché sur la démystification - pas forcément dans une optique négative, mais plutôt au sens de démasquer la superfétation - de l'autre monstre sacré du communisme, car le «bonhomme Lénine» de Malaparte est «un petit bourgeois d'habitudes casanières, perdu dans le tumulte de la révolution comme un bibliothécaire au milieu d'une émeute ${ }^{84} »$. Et même si le Marx de Das Kapital tient tête aux émigrés français à Londres et à leurs femmes, qui lui demandent son appui pour rentrer à Paris combattre Louis-Napoléon - le personnage n'est donc pas dépourvu d'un caractère titanesque, et surtout n'abdique pas de ses principes -, Malaparte décide de nous montrer également ses traits typiquement bourgeois : le père de famille en pantoufles, inquiet de ne pas arriver à « joindre les deux bouts », le mari affectueux d'une femme forte et dévouée. Le personnage malapartien n'est pas non plus à l'abri d'une faiblesse qui peu à peu le submerge et le dépasse ${ }^{85}$, affectant même ses théories et ses analyses - à savoir l'impossibilité, à la base de Das Kapital, qu'en France puissent se réaliser les circonstances de l'avènement d'une révolution prolétarienne, 
d'où l'inutilité que les prolétaires français aillent se faire massacrer sur des barricades dressées contre le coup de main de Louis-Napoléon.

Le tissu des hypotextes de la pièce ne se limite pas aux œuvres de Marx et d'Engels, elles montrent aussi que Malaparte n'a pas évité certains modèles et situations typiquement théâtraux. Ainsi, par exemple, l'on peut supposer que le fait que le «chartiste » Ernest Jones, au premier acte de Das Kapital, ait un rendez-vous le soir même avec une certaine « Lady Windermere ${ }^{86}$ » n'exclut pas un " clin d'œil » à la pièce d'Oscar Wilde, Lady Windermere's Fan, créée en 1892. Parmi les comédies de mœurs de Wilde, en effet, celle-ci est probablement la plus amère, car la satire de la société victorienne s'y mêle à un réel pathos pour les personnages en scène, et est en même temps la plus " ouverte » en termes de dénouement, car le topos de la découverte de liens familiaux cachés ne se produit pas finalement avant le tomber du rideau. Cette teneur énigmatique et non consolatoire est très présente dans Das Kapital, la pièce de Malaparte où la composante "livresque» et la composante "visionnaire » sont d'ailleurs les plus présentes.

Les références hypotextuelles permettent aussi à Malaparte de faire œuvre de glose à l'égard de ses sources, de manière semblable à la stratégie mise en place dans Du côté de chez Proust. Les dialogues de Das Kapital sont ainsi fortement ancrés dans l'interprétation de certains mots-clés. Lorsque deux «Policemen» se rendent chez Marx pour enquêter sur la plainte qui a été déposée contre lui, et d'après laquelle il "soignerait des malades" sans être médecin, le déroulement de la scène se fait décidément métalinguistique, car des discussions s'engagent sur les concepts de " maladie » (au sens physiologique, voire social), de " prolétariat » - une notion qui est à distinguer de celle de "travailleurs", contrairement à ce que font les deux policiers -, d'« étranger ». À un autre moment de la pièce, c'est le concept de "révolution » - et le mot qui va avec - qui fait l'objet d'un débat lexicologique du même type.

Tout comme lorsqu'il se mesure à Proust, Malaparte est bien conscient du fait qu'il est en train de traiter Marx ad usum Francorum. Le dramaturge veut s'adresser à un public et à une classe dirigeante française en particulier, en ne lui épargnant, au cours de la pièce, aucune remarque caustique. Comme c'est souvent le cas chez Malaparte, les considérations sociologiques sur le contingent - la France de l'après-guerre-, évidentes même s'il a recours à un cadre historique, s'absolutisent et tâchent de comprendre et de décrire aussi le "génie du peuple » en question. C'est ainsi qu'au cours de l'acte II de Das Kapital Engels s'écrie: «Les Français aiment les révolutions, mais ils détestent les changements ${ }^{87}$. " Le pessimisme politique de Malaparte émerge tout au long des dialogues, à travers, notamment, les prises de position et les raisonnements de Marx, qui considère que le peuple français a été trahi par une alliance entre les bourgeoisies anglaise et française, qui se seraient fédérées en mandatant Louis-Napoléon pour rétablir l'ordre en France et éviter « les barricades ». Ce pessimisme se teint fréquemment de raillerie. Ainsi : « Les dictateurs bourgeois sont les meilleurs alliés de la révolution prolétarienne ${ }^{88}$. " Aussi, en amont du coup d'État bourgeois y aurait-il la volonté, de la part des États-Unis, d'étendre leur influence sur la politique européenne - ce qui permet à Malaparte d'introduire une thématique qui se réfère à 1948 plus encore qu'à 1851 - car : «Les révolutions de l'Europe ne rapportent qu'aux Américains, c'est connu ${ }^{89}$.» 
Figures 1 et 2. - Personnage de l'Inconnu.

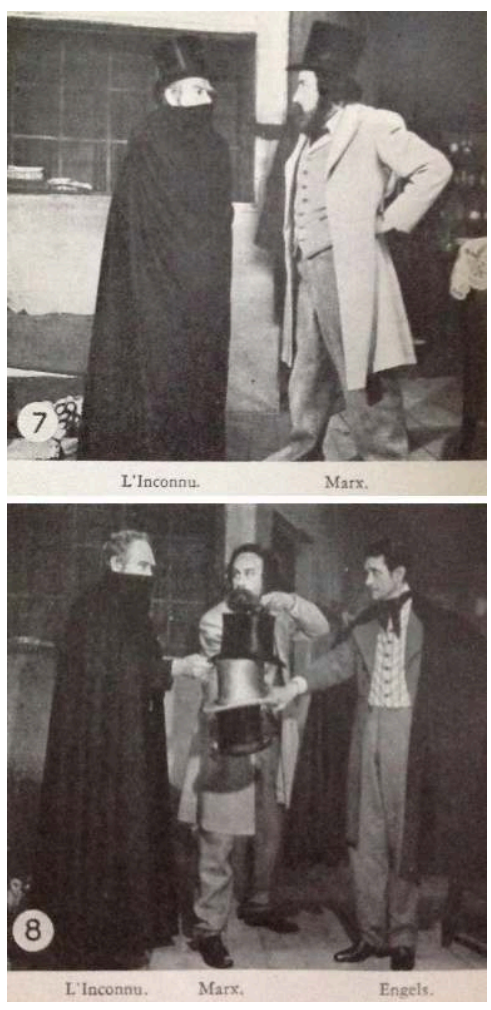

Curzio Malaparte, Das Kapital, dans France Illustration. Supplément théâtral et littéraire, n 36, 11 juin 1949, table hors texte.

Le jugement foncièrement négatif sur la situation française était beaucoup plus poussé dans la prépublication de Das Kapital en revue, par la présence du personnage de l'Inconnu, qui disparaît dans la version en volume. Cette différence montre en même temps l'entité de l'adaptation à laquelle Malaparte soumet son texte avant l'édition qu'il considère comme définitive, dans le but sans doute d'émousser quelque peu les polémiques qui avaient accompagné la création du texte par le théâtre de Paris. Le personnage en question, l'Inconnu (fig. 1 et 2), caractérisé aussi par une apparence qui en souligne le caractère d'Everyman, et pour lequel Malaparte lui-même avait suggéré qu'il pût s'agir de Victor Hugo, a un rôle de premier plan à l'acte III de Das Kapital. Il se présente chez Marx en affirmant venir de la part de Charles Dickens, et il se produit dans un âpre réquisitoire contre la France de "Napoléon le Petit», en citant abondamment le pamphlet de Victor Hugo de 1852 et des vers anti-bonapartistes du poète. La suppression de ce passage dans le texte en volume prive en outre le lecteur de l'un des moments scéniquement les plus réussis de Das Kapital, le " jeu des chapeaux ", dont voici le déroulement quelque peu abrégé :

Karl Marx: [...] (Il ôte son chapeau et le tient suspendu en l'air, à bout de bras.) [...] mettez, je vous prie, votre chapeau sur le mien.

[...] Et toi, Fritz [Friedrich Engels], mets ton chapeau sur celui de Monsieur. Voilà. (Les trois hommes font face à la scène: Karl Marx au milieu, l'Inconnu à sa droite, Friedrich Engels à sa gauche.) Mon chapeau est le prolétariat. Le vôtre, Monsieur, c'est la République. Ton chapeau, Fritz, c'est la Monarchie. [...] Quand je prononcerai le mot "République ", vous placerez rapidement votre chapeau sur celui de notre ami Engels. Et toi, Fritz, quand je prononcerai le mot «Monarchie», tu placeras rapidement ton chapeau sur celui de Monsieur. [...] Nous sommes dans les dernières années du règne de Louis XVI. La Monarchie est en pleine décadence, la révolution 
gronde dans les esprits. Comme vous voyez, Monsieur, l'aristocratie coiffe la bourgeoisie, laquelle coiffe le prolétariat. Attention! 1789! République ! (L'Inconnu place rapidement son chapeau sur celui de Friedrich Engels.) J'entends les pas de Bonaparte Premier Consul, de Napoléon Empereur : Monarchie! (Friedrich place son chapeau sur celui de l'Inconnu.) Île d'Elbe! (L'Inconnu place rapidement son chapeau sur celui de Friedrich Engels.) Non, Monsieur, vous vous trompez. L'Île d'Elbe, c'est toujours la Monarchie, c'est le retour de Louis XVIII. [...] Revenez donc à votre place, Monsieur. Attention! Les Cent Jours! (L'Inconnu et Friedrich Engels changent en même temps de place leurs chapeaux qui se heurtent dans l'air, chacun d'eux s'empressant de mettre son chapeau sur celui de Karl Marx.) Mais non, mais mon, Messieurs! Les Cent Jours, ce n'est pas la République, c'est encore la Monarchie ! [...] Attention! Waterloo, Sainte-Hélène! [...] Ne bougez pas! Juillet 1830 : Louis-Philippe... Ne bougez pas... Février 1848 : République! (L'Inconnu place rapidement son chapeau sur celui de Friedrich Engels.) 2 décembre 1851 ! Louis-Napoléon! Coup d'État ! Dictature ! $\mathrm{Ha} ! \mathrm{Ha} ! \mathrm{Ha}^{90}$ !

56 À travers un jeu de scène qui n'était pas sans introduire un certain dynamisme dans une action dramatique la plupart du temps statique, cette longue citation - qui mérite d'être reportée du fait de la rareté de la prépublication - présente un abrégé de l'histoire moderne de France, du point de vue de la lutte entre les deux formes de gouvernement alternatives et le prolétariat. La démonstration de Marx vise à mettre en avant l'exclusion systématique de ce dernier des vicissitudes politiques françaises.

Dans cette pièce assez prolixe - qui devait durer autour de trois heures ${ }^{91}-$ d'autres moments plus légers sont également présents, tel celui de la «ronde » à laquelle les personnages - y compris les policiers - s'adonnent afin de distraire le petit « Mush » fiévreux, au premier acte ${ }^{92}$. C'est là presque une scène de musical, qui anticipe un intérêt qui sera de plus en plus présent dans la réflexion dramaturgique de Malaparte, et qui émergera en particulier dans son « testament théâtral » Sexophone.

La composante iconoclaste de l'opération dramaturgique de Malaparte est ultérieurement soulignée par le mélange de réalité - les sources qu'il utilise, mais aussi la plupart des personnages de la pièce - et de fiction que l'on trouve dans Das Kapital. Un personnage en ce sens intéressant, de pure invention malapartienne, est celui du secrétaire de Marx, Godson - Gottsohn, en début de projet -, le «fils de Dieu». Ce caractère fictionnel est doté d'une composante surnaturelle qui ressort tout au long de la pièce, dès le début. Par exemple, à l'acte I, Godson montre qu'il est au courant d'une lettre envoyée par Marx à Engels, sans que ni l'un ni l'autre ne la lui aient fait lire ${ }^{93}$. Godson se fait le porte-parole du christianisme, et notamment du protestantisme ${ }^{94}$, face à la douleur de Marx et de Jenny pour la maladie de leur fils, mais aussi face à un Marx désemparé à cause des difficultés que sa vocation révolutionnaire fait vivre à sa famille. Si Jenny tend à se réfugier dans la prière et dans la consolation religieuse, Marx, quant à lui, reste inamovible face au leurre de la religion. Il n'en reste pas moins, cependant, que l'image d'un Marx qui rédige son Capital à l'aide d'un secrétaire-fils de Dieu est pour le moins troublante. En poussant un peu plus loin cette lecture religieuse - et toute la fin de l'acte I est consacrée à une discussion en matière de religion justement -, on pourrait même se demander si, en fin de compte, Marx et Godson ne devraient pas être considérés comme deux avatars différents du divin. Marx serait une divinité juste mais implacable, alors que Godson représenterait plutôt une instance christique, en ce qu'il est capable d'éprouver de la pitiép ${ }^{95}$. Dans les mots de Malaparte lui-même, Godson serait : « una figura [che] si aggira per le stanze, parla e commenta: è 
la rappresentazione di questa parte di Dio nell'uomo, un Gesù Cristo travestito da Charlot [...]. Marx combatte per la giustizia, Cristo-Charlot per la carità ${ }^{96}$ ».

En secondant tour à tour, ou en critiquant la conception des théories qui sont à la base du Capital, Godson met en outre à plusieurs reprises Marx face à lui-même, et en ce sens aussi il agit comme la conscience du personnage. Il affirme par exemple : «On ne peut pas effacer Dieu, Monsieur Marx ${ }^{97}$ !» Et Malaparte à son tour de gloser sur son propre texte : "Nella tragedia personale di Marx io ho inteso rappresentare la tragedia dello spirito umano ${ }^{98}$.» Ou encore : «[...] Gottsohn è in fondo l'esteriorizzazione scenica d'una parte intima dello stesso Marx, come di tutti gli uomini: d'una opposizione che lo stesso Marx avverte, come tutti gli uomini, nella sua coscienza e contro cui lotta ${ }^{99}$. »

Enfin Godson, tout comme le Christ, lorsque Jenny Marx à l'acte II lui demande sèchement qui il est et d'où il vient, répond par la même question, et, de plus, passe à la troisième personne du singulier pour parler de lui-même : "Sait-on qui il est ? Sait-on d'où il vient ${ }^{100}$ ?»

En définitive il est difficile de dire si Das Kapital devrait plutôt être considéré comme une pièce politique ${ }^{101}$, ou bien comme une pièce existentielle, ou encore comme une pièce religieuse, étant donné que les trois composantes interagissent étroitement. La critique française, comme on le verra au prochain paragraphe, ne s'est pourtant guère souciée de cette coprésence, en attribuant presque unanimement à la pièce de Malaparte un caractère politique prédominant.

Une remarque conclusive sur la langue du théâtre français de Malaparte. Celle-ci garde la précision référentielle et la portée fortement connotative de son italien, mais l'écrivain perd néanmoins, dans le passage au français, une partie de sa charge expressive et expressionniste, de son mordant ${ }^{102}$. Le résultat est finalement plus plat sur le plan stylistique, même si les pièces ne manquent pas d'exemples de «style malapartien », comme le dialogue entre Marx et les policiers à l'acte I de Das Kapital, où Marx part d'une anecdote sur Thomas More et la force omnivore des moutons d'Angleterre, renversant et absolutisant ensuite les objections des policiers pour conclure enfin que l'Angleterre, si elle n'est pas mangée par ses moutons, sera néanmoins phagocytée par les machines ${ }^{103}$.

Il semblerait d'ailleurs que Jacques Hébertot (à l'époque le propriétaire du théâtre qui porte encore son nom), après avoir lu Das Kapital en vue de l'inclusion de la pièce dans son affiche de la saison - avant que Malaparte ne décide d'en confier la création au théâtre de Paris - ait suggéré à l'auteur de soumettre le texte à un écrivain francophone «natif » pour le corriger, et que Malaparte ait refusé dédaigneusement le conseil $^{104}$.

64 Giordano Bruni Guerri définit les deux pièces de Malaparte comme deux œuvres "hâtives ", peu muries, et qui auraient mérité d'être mieux pensées ${ }^{105}$, sur le plan linguistique aussi. Ce jugement me semble quelque peu sévère. Évidemment, dans le cas de Das Kapital, mais encore plus dans celui de Du côté de chez Proust, la langue de Malaparte est en quelque sorte "portée » par celle de l'hypotexte déclaré, certains passages des pièces étant truffés de citations et d'autres révélant plutôt leur nature de pastiches proustiens ou marxiens, mais cela n'empêche pas qu'à d'autres moments, la verve linguistique malapartienne émerge, en donnant vie à des scènes très originales et théâtralement réussies. 


\section{Quelques éléments de la fortune et de la réception française des deux pièces portées à la scène}

Il est intéressant d'évoquer en conclusion les questions de la réception de Du côté de chez Proust et de Das Kapital sur la scène parisienne. En dépit du fait que Malaparte ait eu à sa disposition des salles prestigieuses de la capitale et des comédiens et metteurs en scène de renom - Pierre Fresnay, un bon ami de Malaparte ${ }^{106}$, et sa compagne Yvonne Printemps pour l'impromptu, Pierre Dux pour Das Kapital ${ }^{107}$-, les deux pièces ont été mal accueillies par le public et par la critique ${ }^{108}$. Cependant, il semblerait qu'elles soient restées à l'affiche bien plus longtemps que les pièces de D'Annunzio mises en scène en France.

Il est par ailleurs difficile de comprendre la nature précise de la «cabale » dont Malaparte se dit victime de la part du milieu du théâtre parisien, et notamment d'Hébertot, après qu'il a refusé de faire jouer Das Kapital dans son théâtre - une cabale que ses sympathisants confirment. En particulier, est-ce à cause d'un "coup » monté par Hébertot, comme semble le suggérer Malaparte, que les comédiens n'ont pas pu terminer la représentation de Das Kapital lors de la première ${ }^{109}$ ? Et qu'en est-il des négociations entre Malaparte et Hébertot pour la cession exclusive des droits de représentation de Das Kapital ? Enfin, quelle a été la nature exacte des divergences entre les deux ? S'agissait-il de divergences artistiques ou économiques?

Quoi qu'il en soit, tant Du côté de chez Proust que Das Kapital ont suscité de la part de la critique maintes réactions qui vont de l'ironie à l'agacement, et qui nous en disent long sur l'accueil qui pouvait être réservé, en France, au théâtre italien d'expression française, que ce soit celui de D'Annunzio en 1911-1912 ou celui de Malaparte trentecinq ans plus tard.

Commençons par Du côté de chez Proust, à propos duquel un mot qui revient à plusieurs reprises est celui de "profanation ». Le fait de se mesurer avec Proust est un péché d'orgueil pour un écrivain italien, certes, tout comme l'est aussi l'opération dramaturgique en soi, celle d'épitomer la Recherche et d'en faire un impromptu en un acte. Ainsi L'Époque: «On ne réduit pas Proust à un $\operatorname{sketch}^{110}$.» Au lendemain de la création de la pièce, le Figaro organise des entretiens avec des spectateurs "proustiens ", qui pour la plupart critiquent âprement la pièce de Malaparte ${ }^{111}$ - Christian Dior, par exemple, est décrit quittant le théâtre en proie à une crise d'hystérie. Elsa Triolet abonde dans le même sens, en définissant la pièce comme un médiocre « reader's digest » proustien ${ }^{112}$.

Le fait aussi d'avoir fait monter Marcel Proust sur scène n'a guère enthousiasmé la presse française, car il s'agit là aussi d'une désacralisation, d'autant plus que : «[...] M. Malaparte lui [à Marcel Proust] donne l'enveloppe d'un cercleux qui s'essaie à imiter Jean Lorrain et qui récite du Félicien Champsaur ${ }^{113}$ !» On voit ici que le style de l'impromptu aussi fait l'objet de critiques. Le compte rendu négatif de Jean-Jacques Gautier paru dans le Figaro est, en ce sens, célèbre. Gautier trouve le texte de Malaparte assez fade, tout comme le seraient d'ailleurs ses références littéraires et son " proustianisme » (alors même que la critique française avait comparé le style de Kaputt à celui de Proust précisément). Gautier termine son article en affirmant que Du côté de chez Proust, qui est une pièce très obscure, est pour déplaire tant aux proustiens qu'à ceux qui connaissent moins la Recherche - à savoir la majorité des spectateurs -, c'est- 
à-dire ceux qui ont une sorte d'avant-goût ou d'idée reçue sur ce à quoi Proust devrait ressembler ${ }^{114}$. C'est un argument qui sera d'ailleurs remployé pour critiquer Das Kapital, un texte qui devrait - toujours de l'avis de la presse française - susciter l'indignation tant des marxistes que des antimarxistes, tant de ceux qui ont lu Marx que de ceux qui n'ont qu'une idée très vague des idéaux communistes.

Il semblerait que Jean Cocteau et Paul Claudel aient été parmi les rares spectateurs favorablement impressionnés par le travail dramaturgique de Malaparte ${ }^{115}$. Le dramaturge, quant à lui, écrit très souvent aux directeurs des périodiques qui ont publié des comptes rendus au vitriol de la pièce proustienne pour se plaindre du traitement reçu, et pour s'auto-justifier sur ses choix artistiques.

Dans son for intérieur, il en est autrement. En effet, une page inédite citée par Maurizio Serra montre comment Malaparte a interprété ces réactions outrées et ces attaques du public. À nouveau, il a cru à une cabale, qu'il s'explique ainsi :

Il y a dans la haine des invertis sexuels pour ceux qui profanent leur monde secret, quelque chose qui ressemble à une sorte de regret. L'homosexualité qui a eu son idole en Proust n'est plus à la hauteur des temps, elle est désuète, c'est une homosexualité conservatrice de droite. On a observé que les homosexuels conservateurs de droite ont été en très large mesure des collaborateurs des Allemands ${ }^{116}$.

72 Voici typiquement comment Malaparte conçoit les critiques qui lui sont adressées : il les met sur le compte du dépit et de l'indignation du «lobby » homosexuel, qui aurait vu dans le portrait de classe fourni par la pièce de Malaparte un miroir de sa propre déchéance et l'annonce de sa fin... politique, aussi, et de son rôle destructeur à l'égard d'autres groupes sociaux. Il faut néanmoins reconnaître que des remarques sur le rôle des homosexuels dans la désintégration de la société capitaliste étaient déjà présentes dans la didascalie-essai qui précède le texte de Du côté de chez Proust, ce qui atteste qu'une critique du nihilisme politique dont Malaparte accuse les homosexuels pouvait trouver des appuis dans la lecture même de l'impromptu.

D'autre part, la réaction de Malaparte face aux critiques plus décidément à caractère politique qui lui seront adressées pour Das Kapital ne sera pas si différente et, là encore, il les impute à un « lobby ", celles des « italophobes".

Francis Ambrière, critique théâtral pour Opéra et aussi romancier, sera le plus acharné parmi les détracteurs du théâtre de Malaparte. Cette épigramme sur Du côté de chez Proust est d'ailleurs de son fait : "Quand un chien pisse sur une statue, il n'en souille que le socle ${ }^{117}$. » Il s'arme ainsi avant de lancer ensuite sa polémique hargneuse contre Das Kapital, à laquelle Malaparte riposte coup sur coup. Ambrière publie ses articles dans Opéra ${ }^{118}$, donc à la destination d'un lectorat plus spécifiquement " théâtral », et en même temps sur les colonnes du Figaro $^{119}$, et cette double destination se concrétise en un élargissement de la portée de ses arguments : c'est en même temps et l'œuvre, et l'homme qu'il vise. Si déjà dans son premier article le ton est cru, lorsqu'il compare les spectateurs qui ont quitté la salle avant la fin de la représentation de Das Kapital à l'exode des parisiens en 1940 "pendant que les amis de Malaparte mitraillaient les colonnes des réfugiés ", il s'en prend également à Malaparte, "imposteur, fumiste, cerveau brûlé ", il ironise sur sa vie privée, ses maîtresses, et même sur la coupe de ses vêtements. Ambrière accuse en même temps l'œuvre malapartienne de " misogallisme ", attribuant à l'écrivain un pamphlet antifrançais non mieux précisé qui aurait été distribué aux troupes italiennes pendant la guerre, et il lui reproche ses 
poèmes antifrançais de la même époque, surtout Les Morts de Bligny jouent aux cartes. Enfin, il blâme son activité collaborationniste et son rôle ambigu pendant le conflit.

Malaparte répond en accusant Ambrière d'italophobie, mais surtout il provoque son adversaire en duel, et Ambrière accepte. Le duel n'aura finalement pas lieu car les témoins des deux contendants n'arrivent pas à se rencontrer - d'autres versions racontent tour à tour que l'un ou l'autre des deux adversaires se serait retiré1 ${ }^{120}$. On conserve néanmoins un témoignage précieux sur cet épisode, provenant d'un jeune Italien installé à Paris et proche de Malaparte à l'époque de la composition de ses pièces françaises ${ }^{121}$; il aurait été le partenaire d'entraînement de Malaparte en préparation du duel, et il met sur le compte de la lâcheté d'Ambrière et de son inexpérience dans le maniement de l'épée le fait que celui-ci ait fait appel à une sorte de "vice de forme " concernant les délais de tenue des duels pour pouvoir éviter de se battre.

Un exemple du style véhément d'Ambrière dans son jugement de Das Kapital expliquera mieux les raisons de la rage malapartienne :

Ce n'est pas seulement un tissu d'âneries, un cours d'histoire pour arriérés mâtiné $\mathrm{du}$ plus plat mélodrame, c'est une offense à la dignité humaine par tout ce que l'ouvrage contient de bêtise agressive et triomphante, par la manière dont il ravale et salit les grandes idées, les grands esprits, les grandes souffrances auxquels il fait allusion $^{122}$.

Ce qui est sûr, c'est que ce duel manqué ne s'est ensuivi d'aucune réconciliation. Bien plus, les deux hommes continuent de s'injurier par articles interposés. Cette polémique s'élargit aussi dans la mesure où d'autres intellectuels interviennent en se ralliant à l'un ou à l'autre des deux contendants : c'est ainsi que Daniel Halévy prend la défense de Malaparte et risque un duel à son tour. Il convient encore de remarquer à propos de cette situation : il semblerait que la presse italienne ait tenu à ce que la polémique ne n'apaise pas, car bon nombre des articles français - en particulier ceux des adversaires de Malaparte - sont aussi traduits et publiés par des journaux et revues italiens.

Pour le public et les intellectuels français, le Marx mis en scène par Malaparte est "atypique, caricatural pour les marxistes, trop bon et humain pour les antimarxistes ${ }^{123}$ ». Plusieurs gazetiers français n'ont d'ailleurs pas manqué d'inventivité dans leurs critiques. Ainsi, parmi les titres des articles et les boutades trouve-t-on un «Kapital sans intérêt » ou la comparaison entre la pièce malapartienne et « la peine Kapoutale $^{124} »$.

Par rapport à Du côté de chez Proust (mais le jugement est valable aussi pour Das Kapital), Bernard Urbani affirme : «Les Français ont refusé de voir un spectacle ayant comme support le monde réel à partir duquel a travaillé l'artiste ${ }^{125}$. "

80 En tout cas, manifestement, les deux pièces malapartiennes n'ont pas exercé d'influence notable sur le milieu littéraire français, ni sur celui spécifiquement théâtral ${ }^{126}$.

\section{Épitaphe}

$81 \mathrm{Au}$ moment de la mort de Malaparte, ses amis français en écrivent des nécrologies touchantes, et même ses adversaires reviendront sur la vie et l'œuvre de l'écrivain en portant un regard plus clément ou, du moins, plus équilibré. Entre autres, ils reconnaîtront que le "four» de Das Kapital fut injuste, et les polémiques autour de l'œuvre de Malaparte immotivées et contenant une bonne dose de mauvaise foi. Et si le 
Canard enchaîné ironisait, fin 1947, lors du retour de Malaparte en France, sur l'écrivain, en le surnommant « Mâle-Aparte ${ }^{127}$ », Bernard Grasset admettra, par rapport au sort de Das Kapital, que : « Si la pièce était de Sartre, elle serait jouée mille fois ${ }^{128}$ ! »

\section{NOTES}

1. C. Malaparte, Das Kapital et Du côté de chez Proust, Rome, Milan, Aria d'Italia, 1951, p. 357.

2. C'est d'ailleurs en grande partie dans cette perspective que Maurizio Serra (Malaparte. Vies et légendes, Paris, Grasset, 2011) trace son profil biographique de Malaparte écrivain, intellectuel et homme.

3. Malaparte III (1932-1936), E. Ronchi Suckert (éd.), Florence, Ponte alle Grazie, 1992, p. 175.

4. Je suis en effet tenté de faire entrer Don Camalèo dans la série des œuvres «livresques" de Malaparte, même si le fort ancrage de ce roman-essai dans la situation socio-politique italienne en fait un cas-limite. La composante érudite, en effet, même si utilisée de manière parodique et, surtout dans les premiers chapitres, les nombreuses références au Secondo amante di Lucrezia Buti de D'Annunzio, paru en 1924, renforcent l'impression qu'il s'agit d'un livre « fait d'autres livres », et finalement d'un hommage à un certain nombre d'auteurs classiques.

5. M. Serra, Malaparte. Vies et légendes, ouvr. cité, p. 555.

6. Ibid., p. 41-42.

7. Même dans une œuvre comme Maledetti toscani, en fin de compte, émerge chez Malaparte un sentiment de respect et d'admiration sans bornes pour sa terre natale. En ce qui concerne D’Annunzio, des Laudi toscanes au Songe d'un matin de printemps, au Secondo amante di Lucrezia Buti déjà mentionné, et jusqu'au carnet inédit récemment publié sous le titre In Toscana (E. Paccagnini [éd.], Milan, Otto/Novecento, 2013), maintes sont dans l'œuvre de l'écrivain les occasions de célébrer sa terre d'adoption et d'élection.

8. Le célèbre Febo de Malaparte d'un côté, et la monumentalisation de la cynophilie dannuntienne à travers la construction d'un cimetière de ses chiens au Vittoriale d'un autre côté.

9. M. Serra, Malaparte. Vies et légendes, ouvr. cité, p. 556.

10. Eugenio Montale, «L'arcitaliano », Il Corriere della Sera, 20 juillet 1957.

11. Sur toutes ces questions, et pour un encadrement général de la problématique de Malaparte et le théâtre, voir G. Panella, La vocazione sospesa. Curzio Malaparte autore teatrale e regista, Rome, Fermenti, 2013.

12. Malaparte tiendra une rubrique de critique dramatique pour l'hebdomadaire Aux Écoutes: voir Malaparte VIII (1948-1949), E. Ronchi Suckert (éd.), Florence, Ponte alle Grazie, 1994, passim.

13. L'on connaît un autre court fragment théâtral français de Malaparte, Les Joyeux compères de Yalta : cf. Malaparte IX (1950-1951), E. Ronchi Suckert (éd.), Florence, Ponte alle Grazie, 1994, p. 377-380.

14. Il semblerait que cette pièce ait eu un succès international, s'il faut croire Malaparte, qui, dans un article du 13 octobre 1955, parle d'une création de Anche le donne hanno perso la guerra à Buenos Aires dans deux théâtres simultanément en juillet 1955, en faisant remarquer que les représentations se suivent encore à l'automne de la même année. Cf. Malaparte X (1952-1954), E. Ronchi Suckert (éd.), Florence, Ponte alle Grazie, 1995, passim. Celui des femmes qui ont perdu (ou gagné) la guerre est un projet que Malaparte a mûri entre France et Italie ; il le commence d'abord fin 1951 sous la forme d'un «burlesque » en trois actes, également inachevé, où l'on 
trouve d'ailleurs, probablement en hommage à Proust, un « Alberto » qui devient « Albertina ». Cf. ibid., p. 225-281, et M. Serra, Malaparte. Vies et légendes, ouvr. cité, p. 467. Il s'agit donc d'un « chantier » postérieur par rapport aux deux ébauches dont il est question dans ce paragraphe. Ce projet culminera en 1954 avec la pièce Anche le donne hanno perso la guerra, créée au théâtre La Fenice de Venise le 11 août 1954 dans le cadre du Festival Internazionale di Teatro della Biennale et publiée la même année (Bologne, Cappelli). Lorsqu'on lit le long passage sur les femmes et la Deuxième Guerre que l'on trouve dans Les Amazones, on se rend compte que cette thématique a longtemps hanté Malaparte. Les femmes des Amazones auraient, en fait, été les seules perdantes à l'issue du conflit. Mais c'est là une idée qui, comme c'est fréquemment le cas chez Malaparte, se prête à maints renversements. À travers cette défaite, en effet, les femmes auraient été les seules vraies gagnantes de la guerre.

15. On peut lire les fragments des Amazones dans Malaparte VII (1946-1947), E. Ronchi Suckert (éd.), Florence, Ponte alle Grazie, 1993, p. 737-777, en particulier p. 765-766.

16. Ibid., p. 754. Ici et ailleurs, j'ai pris soin de corriger les très nombreuses coquilles dans les textes français des volumes des inédits sans le signaler dans l'article. Elles sont sans doute dues à une inadvertance de l'éditeur plus qu'à Malaparte lui-même.

17. Ibid., p. 755.

18. Ibid., p. 759.

19. Ibid.

20. Ibid. Sans qu'on puisse indiquer de référence précise à Musset - sauf sur le plan de l'imaginaire d'une George Sand en "Amazone ", sans doute - il faut considérer que l'écrivain romantique compte parmi les principaux modèles théâtraux de Malaparte, aussi bien pour le sens de la repartie qu'on trouve dans Du côté de chez Proust, que pour le choix même du sous-genre pour cette dernière pièce, celui de l'impromptu, une forme qui est très proche des " proverbes » de Musset.

21. Ibid., p. 757.

22. Ibid., p. 758. D'ailleurs, la guerre est aussi conçue comme un match sportif (cf. le discours du Roi à l'acte II, ibid., p. 765). Vice versa, les sports - la boxe et le rugby en particulier - sont assimilés à la guerre. Ainsi le rugby est-il défini « guerre totalitaire » (ibid., p. 768).

23. Ibid., p. 763-764. L'astérisque qui suit la référence à l'Arlequin aurait probablement été remplacé par Malaparte par l'indication d'un artiste moderne (car Alessandro Magnasco était actif entre la deuxième moitié $d u X I_{I}{ }^{e}$ et la première $d u X V I I I^{e}$ siècle), s'il n'avait pas laissé la pièce inachevée. Si la chronologie relative que j'ai pu reconstruire est exacte, il s'agit en même temps d'une ébauche de la didascalie initiale de Du côté de chez Proust, comme il semble évident du fait que certains noms de peintres (De Chirico) et de sujets de tableaux (l'Arlequin) sont présents dans les deux textes. Cf. C. Malaparte, Das Kapital et Du côté de chez Proust, ouvr. cité, p. 11 et suiv.

24. Cf. Malaparte VIII (1948-1949), ouvr. cité, p. 675-707.

25. Il s'agit d'une pièce dont la situation textuelle est particulièrement complexe. Le volume des inédits contient en effet un premier acte qu'on peut considérer complet - mais qui aurait sans doute nécessité une révision de la part de l'écrivain - et des scènes éparses du deuxième et du troisième acte. De plus, la lecture est ultérieurement compliquée par le fait que l'édition en question mélange des passages d'une "première rédaction" et d'autres appartenant à une "deuxième", sans que les circonstances de composition de l'une et de l'autre ne soient clairement explicitées. D'après ce que j'ai pu en déduire, l'idée d'une pièce située à Sienne s'impose à Malaparte dans un deuxième temps, une fois fixé le "nœud dramatique ", qui est constitué par le patriotisme des femmes d'une ville assiégée qui se prostituent aux assiégeants en échange de vivres pour leurs compatriotes masculins. Le cadre dans lequel devait se situer l'action dramatique aurait d'abord dû être celui du Mexique ou d'une ville d'Amérique centrale ou méridionale, toujours assiégée par les Espagnols, comme c'est le cas pour la bourgade de Montelsa qui est enfin retenue comme cadre. 
26. Les deux seules pièces françaises achevées étaient en effet à l'affiche pendant que Malaparte se consacrait aux Femmes de Sienne (ibid., p. 675).

27. Cf. infra.

28. Ibid., p. 676 et p. 679.

29. Cf. à ce propos la scène entre Messer Toso et Béatrice dans l'acte II (ibid., p. 700-701), qui consiste en un long quiproquo sur la « fatigue » endurée par Béatrice après une nuit passée dans le campement espagnol.

30. Une bourgade qu'on imagine donc située dans la Valdelsa, même s'il s'agit d'un toponyme d'invention malapartienne.

31. Ibid., p. 676.

32. Et n'importe où, ajouterais-je, compte tenu de la portée universelle de l'apologue théâtral de Malaparte.

33. Ibid., p. 680.

34. Ibid., p. 693.

35. C. Malaparte, Das Kapital et Du côté de chez Proust, ouvr. cité, p. 224.

36. Sur la genèse des deux œuvres et sur leur réception, je renvoie au riche dossier - même si assez chaotique et difficilement lisible - recueilli dans Malaparte VIII (1948-1949), ouvr. cité, p. 9 et suiv.

37. Cf. en effet les quelques lettres que lui adresse Guy Tosi et qu'on peut lire dans ibid., passim. Tosi, qui était à l'époque directeur littéraire des éditions Denoël - en plus d'être un célèbre italophile et italianisant - suggère à Malaparte des modifications et des coupures dans le texte de Das Kapital, par rapport auxquelles cf. infra, n. 92.

38. Les parties chantées n'ont pas été composées par Malaparte. Yvonne Printemps, la comédienne qui jouait le personnage de Rachel Quand du Seigneur, aurait performé sur scène des musiques célèbres de l'époque. Cf. à ce propos M. Tanant, «L'Italie interdite. Malaparte et le théâtre ", Chroniques italiennes, $\mathrm{n}^{\circ} 44,1995$, p. 1-13, en particulier p. 3 ; cet article a été repris sous le même titre dans Non dimenticarsi di Proust. Declinazioni di un mito nella cultura moderna, A. Dolfi (dir.), Florence, Firenze University Press, 2014, p. 479-489.

39. Ibid., p. 6. M. Tanant cite à ce propos l'autobiographie de Pierre Fresnay, qui parle d'une vingtaine de représentations. G. B. Guerri, dans L'arcitaliano, Milan, Bompiani, 2009 [1980], p. 234, évoque plutôt une centaine de représentations avant que la pièce ne soit retirée de l'affiche, ce qui semblerait plus plausible du fait que Du côté de chez Proust était combiné avec une pièce d'un auteur à succès - mais rien n'empêche d'ailleurs que la direction du théâtre ait décidé de remplacer l'acte unique de Malaparte par une suite plus en accord avec les goûts du public. Il faut d'ailleurs considérer aussi que ce nombre bien plus élevé de représentations est suggéré par Malaparte lui-même dans une lettre à Orfeo Tamburi, citée par J.-C. Thiriet, Paris la haine, dans Curzio Malaparte. Il narratore, il politologo, il cittadino di Prato e dell'Europa, R. Barilli et V. Baroncelli (dir.), Naples, CUEN, 2000, p. 315-325, en particulier p. 315. La sœur de Malaparte, quant à elle, sans citer de source précise, évoque le chiffre de « quasi 300 » (Malaparte VIII, ouvr. cité, p. 9).

40. C. Malaparte, Du côté de chez Proust, dans Opéra. Hebdomadaire de théâtre, du cinéma, des lettres et des arts. Supplément théâtral, $\mathrm{n}^{\circ} 1$, mars 1949, p. 27-41.

41. E. Ferrieri, Con Proust, créé par La Compagnia del Convegno le 25 avril 1958. Le tapuscrit inédit est actuellement conservé aux Archives d'État de Milan, tout comme la traduction italienne réalisée par Ferrieri de Das Kapital, également inédite. Das Kapital a été aussi traduit par M. Maranzana et publié chez Mondadori en 1980. La pièce a été jouée en Italie, à Trieste, en 1981.

42. Ironie du sort, Roussin était à l'époque l'un des grands auteurs de théâtre bourgeois à la mode, alors que l'impromptu de Malaparte jette un regard très cruel sur la bourgeoisie de la génération d'avant la Première Guerre.

43. Comme il résulte d'une lettre fort intéressante de Pierre Dux à Malaparte, écrite le 9 octobre 1948 - cf. Malaparte VIII, ouvr. cité, p. 203-207 -, Dux avait très à cœur la création de Das Kapital, 
qu'il jugeait une excellente pièce. Il se montre quelque peu soulagé par le refus - ou du moins les hésitations - du confrère, car il juge que «[...] la pièce se suffit à elle-même, [...] il n'est pas essentiel de réunir une distribution tapageuse [...]» (ibid., p. 205). Pour la distribution complète des rôles, cf. ibid., p. 335.

44. Cf. ibid., p. 9 et passim.

45. C. Malaparte, Das Kapital, dans France-Illustration. Supplément théâtral et littéraire, $\mathrm{n}^{\circ}$ 36, 11 juin 1949. Sans que j'aie procédé à une collation complète entre cette prépublication et l'édition en volume, je signalerai néanmoins dans les pages qui suivent une modification importante subie par le deuxième texte - pour des raisons qui sont difficiles à déterminer, mais qui ont sans doute à voir avec la volonté, de la part de Malaparte, d'émousser les polémiques nées autour de la pièce. 46. L'acte unique de Malaparte n'est pas à confondre avec celui, homonyme, de Jacques Sereys de la Comédie-Française, créé en 2005 au théâtre Montparnasse. La pièce de Sereys est plus clairement une adaptation du texte proustien, notamment de Du côté de chez Swann. Sereys a plus récemment écrit une sorte de suite de ce texte, intitulée À la recherche du temps Charlus, créée en 2011.

47. À quelques années de distance, l'hommage à ce type de tradition sera proposé par Malaparte de manière encore plus poussée dans Sexophone, une pièce conçue pour être aussi jouée par Malaparte lui-même : cf. Malaparte XI (1955), E. Ronchi Suckert (éd.), Florence, Ponte alle Grazie, 1996, passim.

48. Il s'agit de Voltaire dans Sodoma e Gomorra. Sans considérer le titre du recueil, d'ailleurs, qui est lui aussi évidemment proustien, plus que biblique.

49. C. Malaparte, Kaputt, dans Id., Opere scelte, L. Martellini (éd.), Milan, Mondadori («I Meridiani »), 1997, p. 461-463.

50. Cf. en particulier ibid., p. 593, 805 et 903. Pour une lecture approfondie du «proustianisme » de Malaparte, et de Kaputt en particulier, je renvoie à J.-C. Thiriet, «Kaputt, dalla parte di Guermantes ", dans «La Bourses des idées du monde». Malaparte e la Francia, Atti del Convegno internazionale di studi su Curzio Malaparte (Prato-Firenze, 8-9 novembre 2007), M. Grassi (dir.), Florence, Olschki, 2008, p. 109-123.

51. C. Malaparte, Das Kapital et Du côté de chez Proust, ouvr. cité, p. 11.

52. Ibid.

53. Cf. ibid., p. 12.

54. Ibid., p. 13-14.

55. Ibid., p. 15.

56. Ibid., p. 16. Tout comme Rachel Quand du Seigneur sera à maintes reprises définie comme « littéraire » dans la Recherche, notamment dans la première partie du Côté de Guermantes.

57. Ibid., p. 16.

58. Ibid., p. 18.

59. Ibid., p. 19.

60. Ibid.

61. Et pour Saint-Loup lui-même, dont les premières apparitions dans le cycle proustien sont fortement marquées par la sympathie que l'aristocrate éprouve pour le socialisme.

62. Cf. infra pour un tout autre point de vue exprimé par Malaparte lors de la défense qu'il mettra en acte face à la « cabale » réservée à ses pièces parisiennes. Dans cette autre circonstance, il s'en prendra en effet à la nature politique foncièrement conservatrice et «de droite " des homosexuels.

63. Ibid., p. 21.

64. Cf. ibid.

65. Ibid., p. 28.

66. Ibid.

67. Ibid., p. 22. 
68. Ibid., p. 20.

69. Cf. respectivement M. Proust, À la recherche du temps perdu, J.-Y. Tadié (dir.), quatre volumes, Paris, Gallimard (« Bibliothèque de La Pléiade »), 1987-1989, I, p. 566-567 ; II, p. 451 et suiv. ; IV, p. 572-573.

70. B. Urbani, dans l'article «Du côté de chez Proust de Malaparte ", dans La tentation théâtrale des romanciers, P. Chardin et al. (dir.), Paris, SEDES, 2002, p. 94-111, parle à juste titre d'un " théâtre critique original » (p. 110).

71. M. Proust, À la recherche du temps perdu, ouvr. cité, II, p. 455.

72. Ibid., p. 456.

73. Ibid., p. 459.

74. C. Malaparte, Das Kapital et Du côté de chez Proust, ouvr. cité, p. 25.

75. Sur ces aspects, cf. aussi J.-C. Thiriet, « Rachel de Proust à Malaparte », Novecento, ${ }^{\circ}{ }^{\circ} 22,1999$, p. $155-165$.

76. M. Serra (Malaparte. Vies et légendes, ouvr. cité, p. 459) définit par contre Du côté de chez Proust une pièce «sans dénouement véritable ».

77. C. Malaparte, Das Kapital et Du côté de chez Proust, ouvr. cité, p. 11.

78. M. Serra, Malaparte. Vies et légendes, ouvr. cité, p. 457.

79. Je nuance ici quelque peu la remarque de M. Serra, qui considère Du côté de chez Proust comme une œuvre dépourvue d'érotisme (ibid., p. 56).

80. Cf. ibid., p. 460.

81. M. Tanant, « L'Italie interdite », art. cité, p. 7.

82. C. Malaparte, Das Kapital et Du côté de chez Proust, ouvr. cité, p. 125.

83. Die achtzehnte Brumaire des Louis Napoléon, recueil d'articles paru en 1852.

84. C. Malaparte, Prologue à la traduction française du Bonhomme Lénine (Paris, Grasset, 1932), p. IX.

85. Comme d'ailleurs l'avait fait remarquer Pierre Dux dans la lettre déjà citée (Malaparte VIII, ouvr. cité, p. 205-206).

86. Cf. C. Malaparte, Das Kapital et Du côté de chez Proust, ouvr. cité, p. 154.

87. Engels dans l'acte II (ibid., p. 234).

88. Toujours Engels dans l'acte II (ibid., p. 227).

89. Mme Bertaud dans l'acte I (ibid., p. 136).

90. C. Malaparte, Das Kapital, dans France-Illustration, ouvr. cité, p. 27. J'ai harmonisé l'emploi des majuscules dans la citation.

91. Lettre de Guy Tosi à Malaparte du 15 septembre 1948 (Malaparte VIII, ouvr. cité, p. 185), dans laquelle l'éditeur et italianiste parle de ses impressions sur le troisième acte de la pièce : «Il est digne des deux autres, presque trop riche en effets scéniques. Le dialogue gagnerait à être un peu plus sobre et moins abstrait par endroits. »

92. C. Malaparte, Das Kapital et Du côté de chez Proust, ouvr. cité, p. 174.

93. À l'acte I (ibid., p. 204-205).

94. Dans une lettre du 4 décembre 1948 à la traductrice anglaise Kitty Black (Malaparte VIII, ouvr. cité, p. 171-173), Malaparte affirme : «[...] ça aurait été ridicule, et arbitraire, de faire agir Marx et les autres comme dans une pièce réaliste » (p. 172). Et plus loin il souligne que la morale de la pièce est à considérer une morale protestante (ibid., p. 173). Un critique d'exception comme Salvatore Quasimodo («Carlo Marx resuscitato da Malaparte », Il Tempo, 5-12 février 1949, ibid., p. 375-376) définit Godson « ossesso » et « mistico » « più che cristiano » (p. 376).

95. M. Serra (Malaparte. Vies et légendes, ouvr. cité, p.460) parle, quant à lui, d'un horizon théologique vétérotestamentaire dans lequel se meut Marx, et d'une éthique plutôt néotestamentaire dans le cas de Godson. Dans Kaputt, Malaparte lui-même avait déjà parlé de la tentative, de la part de Marx, de tuer Dieu, et avait mis côte à côte l'Évangile et Das Kapital (C. Malaparte, Opere scelte, ouvr. cité, p. 724). 
96. P. Zannoni, «Il "Quarantotto" di Malaparte» (interview), L'Avvenire d'Italia, 8 janvier 1948, dans Malaparte VIII, ouvr. cité, p. 17-18, réf. p. 17.

97. Godson dans l'acte II (C. Malaparte, Das Kapital et Du côté de chez Proust, ouvr. cité, p. 245).

98. S. D’Amico, "Vanno in scena a Parigi due commedie di Malaparte» (interview), Il Tempo, 25 octobre 1948, dans Malaparte VIII, ouvr. cité, p. 227-229, ici p. 228.

99. Ibid., p. 229.

100. C. Malaparte, Das Kapital et Du côté de chez Proust, ouvr. cité, p. 255.

101. Pierre Dux, dans la lettre déjà citée (Malaparte VIII, ouvr. cité, p. 206), conseillait à Malaparte de changer le titre de la pièce, voire d'y ajouter un sous-titre, qui aurait pu indiquer aux spectateurs qu'il ne s'agissait pas d'une pièce politique.

102. Cf. aussi le jugement de M. Serra : «Son français, souvent fleuri et trop précieux, ne retient pas l'extraordinaire plasticité de son italien; mais il est généralement précis (malgré les erreurs d'orthographe et les barbarismes qui émaillent ses manuscrits) [...]. » (Malaparte. Vies et légendes, ouvr. cité, p. 463.) Je pense néanmoins que les « erreurs » et les «barbarismes » auxquels Serra fait référence doivent en réalité - du moins en partie - être attribués à la transcription des inédits.

103. C. Malaparte, Das Kapital, ouvr. cité et Du côté de chez Proust, ouvr. cité, p. 164-165.

104. Je prends ce détail de G. B. Guerri, L'arcitaliano, ouvr. cité, p. 234. Guerri cite à son tour le passage d'un article de Domenico Bartoli publié dans Stampa Sera du 21 septembre 1948 (ibid., p. 307, n. 24). D'autres sources de l'époque attestent qu'Hébertot aurait voulu le réécrire luimême (cf. Malaparte VIII, ouvr. cité, p. 9 et, dans un article d'Etta Comito paru dans le Corriere di Napoli le 29 octobre 1948, ibid., p. 236), sans doute, dans ce cas, pour que Malaparte partageât les droits d'auteur avec lui (ibid., p. 330).

105. G. B. Guerri, L'arcitaliano, ouvr. cité, en particulier p. 233.

106. En 1955 encore, Malaparte songea à Pierre Fresnay pour incarner le Mahomet d'un projet de scénario auquel il travaillait : cf. Malaparte XI, ouvr. cité, p. 137.

107. Michel Simon hésita longtemps avant de décliner la proposition de jouer le personnage de Karl Marx.

108. C'est du moins l'impression que j'ai eue en lisant les dossiers de presse. Ce qui me semble néanmoins digne de mention est que les critiques envoyés par la presse italienne pour assister aux représentations des pièces de Malaparte tendent à donner des avis beaucoup plus favorables, sans doute pour une question de fierté nationale -même si Malaparte n'était pas particulièrement aimé en Italie -, et que même les échos italiens des comptes rendus parus en France renversent souvent les jugements d'outre-Alpes. Ainsi, les fiascos malapartiens deviennent aisément des «successi » ou même des «trionfi ». Se faire une idée de l'accueil du public - y compris intellectuel - français est également compliqué à cause de la grande hétérogénéité de ce type de sources, et des fréquentes contradictions qu'on y trouve. Par exemple, lorsque certains chroniqueurs français peignent les réactions outrées de certains spectateurs "illustres ", ces mêmes spectateurs écrivent peu de jours après à Malaparte pour le féliciter de la réussite de sa pièce (Malaparte VIII, ouvr. cité, passim). Il peut s'agir bien évidemment de mauvaise conscience ou d'hypocrisie, mais il me semble que ces réactions contradictoires doivent nuancer quelque peu l'idée d'un échec.

109. Comme d'habitude sans mentionner de source, Edda Ronchi Suckert fait référence à une « incursion de banlieusards» soudoyés par Hébertot (ibid., p. 9 et 214-215). Cf. néanmoins le témoignage du comédien Pierre Dux (Vive le théâtre, Paris, Stock, 1984), cité par M. Tanant, «L'Italie interdite ", art. cité, p. 9, n. 17.

110. François de Roux, "Les Spectacles de la Michodière ", L'Époque, 24 novembre 1948, dans Malaparte VIII, ouvr. cité, p. 252.

111. "Curzio Malaparte a-t-il trahi Marcel Proust?", Le Figaro, 24 novembre 1948, dans ibid., p. 253. 
112. Cités par M. Tanant, «L'Italie interdite », art. cité, passim.

113. M. Favalelli, «Au Théâtre de la Michodière Les Eufs de l'autruche et Du côté de chez Proust ", L'Intransigeant, 24 novembre 1948, dans Malaparte VIII, ouvr. cité, p. 253-254, ici p. 254.

114. Toujours cités par M. Tanant, «L'Italie interdite », art. cité, passim.

115. Cf. Malaparte VIII, ouvr. cité, passim, en particulier p. 337.

116. M. Serra, Malaparte. Vies et légendes, ouvr. cité, p. 459-460. La version italienne est dans Malaparte VIII, ouvr. cité, p. 281 : « V’è nell'odio degli invertiti sessuali per coloro che profanano il loro mondo segreto, qualcosa che assomiglia a una specie di rammarico. [...] L'omosessualità, che ha avuto l'idolo in un Proust, non è più all'altezza dei tempi, è antiquata, è un'omosessualità conservatrice, di destra. È stato osservato da molti che gli omosessuali conservatori, di destra, erano stati in grandissima parte, quale più quale meno, dei collaboratori dei tedeschi. »

117. Cf. M. Tanant, «L'Italie interdite ", art. cité.

118. Cf. déjà F. Ambrière, « Das Kapital au Théâtre de Paris », Opéra, 2 février 1949. Ironie du sort, l'article d'Ambrière est publié par le même journal dont le supplément théâtral publiera, le mois suivant, Du côté de chez Proust. Les pièces principales de cette polémique, qu'on peut lire désormais dans le huitième tome des inédits de Mapalarte (ouvr. cité), dont elles occupent une bonne moitié, sont mentionnées aussi par M. Serra, Malaparte. Vies et légendes, ouvr. cité, p. 466, n. 1.

119. F. Ambrière, « Monsieur Caméléon », Le Figaro littéraire, 5 février 1949.

120. G. B. Guerri, L'arcitaliano, ouvr. cité, p. 234-235, et M. Tanant, «L'Italie interdite », art. cité, p. 9, semblent par exemple pencher pour un désistement de la part de Malaparte.

121. Les souvenirs de Malaparte d'Onofrio Solari Bozzi peuvent être lus en appendice à M. Serra, Malaparte. Vies et légendes, ouvr. cité, p. 572-577.

122. F. Ambrière, «Das Kapital au Théâtre de Paris », Opéra, 2 février 1949, dans Malaparte VIII, ouvr. cité, p. 358-360, ici p. 358-359.

123. J.-C. Thiriet, Paris la haine, art. cité, p. 325 (je traduis).

124. Malaparte VIII, ouvr. cité, p. 403.

125. Cf. aussi B. Urbani, " Du côté de chez Proust de Malaparte », ouvr. cité, p. 109.

126. La même chose peut être dite de l'Italie. On ne peut pas vraiment affirmer que les pièces malapartiennes ont eu un impact sur la culture de l'époque. À une période beaucoup plus récente, par contre, Du côté de chez Proust a sans doute été parmi les sources du récit de Luigi Malerba, Tutta colpa di Proust, tiré du recueil Testa d'argento (Milan, Mondadori, 1988).

127. Cité par J.-C. Thiriet, Paris la haine, art. cité, p. 324.

128. Ce jugement est rapporté par Daniel Halévy et contenu dans la préface à la traduction française faite par Halévy lui-même de Anche le donne hanno perso la guerra. Je cite depuis M. Serra, Malaparte. Vies et légendes, ouvr. cité, p. 463.

\section{RÉSUMÉS}

La présente contribution se propose d'étudier la production dramatique française de Curzio Malaparte aussi bien dans sa composante «livresque ", intertextuelle, que dans sa dimension plutôt « visionnaire ", d'appropriation des sources. Mon hypothèse de travail, vérifiée dans les projets dramatiques inachevés, dans les pièces portées à la scène et dans l'histoire textuelle de ces dernières, consiste en une lecture du théâtre français de Malaparte comme moment privilégié 
de prise de distance, de la part du dramaturge, par rapport à son engagement politique. La dernière section de cet essai est consacrée aux réactions de la presse française face aux mises en scène parisiennes de Malaparte. Mon analyse cherche à éclaircir les raisons implicites de cette réception presque unanimement négative.

Il presente contributo si propone di studiare la produzione drammatica francese di Curzio Malaparte sotto il duplice aspetto della componente 'libresca', intertestuale, e di quella 'visionaria', di appropriazione delle fonti. L'ipotesi di lavoro, verificata tanto nei progetti teatrali incompiuti, quanto nelle pièce portate alla scena, quanto, infine, nella storia testuale di queste ultime, consiste in una lettura del teatro francese di Malaparte come momento privilegiato di un certo distacco dalla militanza politica da parte del drammaturgo. L'ultima sezione del saggio è dedicata alle reazioni - per lo più negative - della stampa francese a fronte delle messinscene parigine di Malaparte, cercando di mettere in evidenza le ragioni implicite di una condanna pressoché unanime.

This paper focuses on the French plays written by Malaparte. Its aim is to discuss both their "bookish"-i.e. intertextual-dimension, and their "visionary" one, which has to do with Malaparte's appropriation of his sources. The essay analyses Malaparte's dramatic sketches and drafts, the plays which have been staged and their textual history. My point is that Malaparte's playwriting can be read as a moment of disengagement from his constant political commitment. The last section of the paper explores the reaction of the French newspapers to the Parisian staging of Malaparte's plays and tries to find out the implicit reasons of a quasi-universally negative response of the critics.

\section{INDEX}

Parole chiave : Malaparte, teatro francese, Du côté de chez Proust, Das Kapital, ricezione Keywords : Malaparte, French theatre, Du côté de chez Proust, Das Kapital, critical response Mots-clés : Malaparte, théâtre français, Du côté de chez Proust, Das Kapital, réception

\section{AUTEUR}

\section{FILIPPO FONIO}

Université Grenoble Alpes 